\title{
Search for an excited lepton that decays via a contact interaction to a lepton and two jets in proton-proton collisions at $\sqrt{s}=13 \mathrm{TeV}$
}

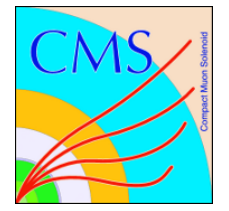

\section{The CMS collaboration}

E-mail: cms-publication-committee-chair@cern.ch

ABSTRACT: Results are presented from a search for events containing an excited lepton (electron or muon) produced in association with an ordinary lepton of the same flavor and decaying to a lepton and two hadronic jets. Both the production and the decay of the excited leptons are assumed to occur via a contact interaction with a characteristic energy scale $\Lambda$. The branching fraction for the decay mode under study increases with the mass of the excited lepton and is the most sensitive channel for very heavy excited leptons. The analysis uses a sample of proton-proton collisions collected by the CMS experiment at the LHC at $\sqrt{s}=13 \mathrm{TeV}$, corresponding to an integrated luminosity of $77.4 \mathrm{fb}^{-1}$. The four-body invariant mass of the two lepton plus two jet system is used as the primary discriminating variable. No significant excess of events beyond the expectation for standard model processes is observed. Assuming that $\Lambda$ is equal to the mass of the excited leptons, excited electrons and muons with masses below 5.6 and $5.7 \mathrm{TeV}$, respectively, are excluded at $95 \%$ confidence level. These are the best limits to date.

KEYwords: Beyond Standard Model, Hadron-Hadron scattering (experiments)

ARXiv EPrint: 2001.04521 


\section{Contents}

1 Introduction 1

2 The CMS detector $\quad 2$

3 Model and signal simulation $\quad 3$

4 Event reconstruction $\quad 5$

$\begin{array}{llr}5 & \text { Backgrounds } & 6\end{array}$

$\begin{array}{lll}6 & \text { Event selection and validation regions } & 7\end{array}$

$\begin{array}{lll}7 & \text { Systematic uncertainties } & 10\end{array}$

$\begin{array}{llr}8 & \text { Results } & 11\end{array}$

$\begin{array}{lll}9 & \text { Summary } & 15\end{array}$

$\begin{array}{lr}\text { The CMS collaboration } & 20\end{array}$

\section{Introduction}

The standard model (SM) of particle physics accurately describes a broad range of observations, but it does not provide an explanation for many of its own features. Among the most prominent of these features is the existence of three fermion generations, encompassing both leptons and quarks. Attempts to explain the observed generation structure have led to a class of models postulating that quarks and leptons are composite objects that consist of more fundamental constituents [1-9]. In these models, the fundamental constituents are bound by an asymptotically free gauge interaction that becomes strong below a characteristic scale $\Lambda$. Such compositeness models predict the existence of excited states of quarks $\left(\mathrm{q}^{*}\right)$ and leptons $\left(\ell^{*}\right)$ at the characteristic scale of the new binding interaction. Since these excited fermions couple to ordinary SM fermions, they could be produced via contact interactions (CI) in collider experiments, with subsequent decays to SM fermions through the gauge interactions (GIs), via the emission of a W or Z boson or a photon $(\gamma)$, or via a CI to other fermions.

Searches at the CERN LEP [10-13], DESY HERA [14], and the Fermilab Tevatron [1518] have found no evidence for excited leptons. At the CERN Large Hadron Collider (LHC), previous searches performed by the ATLAS [19-21] and CMS collaborations [22, 23] have also found no evidence of excited leptons. The maximum $\Lambda$ value excluded experimentally 


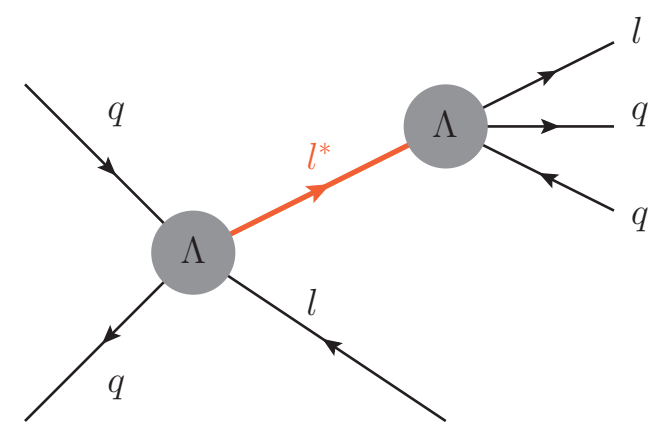

Figure 1. Feynman diagram for the production of an excited lepton in association with an SM lepton in a hadron collider. The excited lepton decays via a contact interaction to one SM lepton and two resolved jets, which result from the hadronization of the quarks.

corresponds to $25 \mathrm{TeV}$ (for $M_{\ell^{*}} \approx 1 \mathrm{TeV}$ ) from a recent CMS $\ell \ell \gamma$ analysis using protonproton (pp) collision data at $\sqrt{s}=13 \mathrm{TeV}$, corresponding to an integrated luminosity of $35.9 \mathrm{fb}^{-1}[24]$. The same analysis excluded excited electrons and muons with masses below 3.7 and $3.8 \mathrm{TeV}$, respectively, for $M_{\ell^{*}}=\Lambda$.

In this paper, a search for excited leptons $\left(\mathrm{e}^{*}\right.$ and $\left.\mu^{*}\right)$ is presented. This analysis focuses on the production of two same-flavor leptons of which one is excited and the other one corresponds to an SM lepton. The excited lepton is assumed to decay subsequently via a CI to an SM lepton of the same flavor and a quark pair, yielding two jets. The process is illustrated in figure 1.

The data used for this analysis were recorded with the CMS detector in pp collisions at $\sqrt{s}=13 \mathrm{TeV}$ during 2016 and 2017 and correspond to integrated luminosities of 35.9 and $41.5 \mathrm{fb}^{-1}$, respectively, for a total of $77.4 \mathrm{fb}^{-1}$.

\section{The CMS detector}

The central feature of the CMS apparatus is a superconducting solenoid of $6 \mathrm{~m}$ internal diameter, providing a magnetic field of $3.8 \mathrm{~T}$. Within the solenoid volume are a silicon pixel and strip tracker, a lead tungstate crystal electromagnetic calorimeter (ECAL), and a brass and scintillator hadron calorimeter (HCAL), each composed of a barrel and two endcap sections. Extensive forward calorimetry complements the coverage provided by the barrel and endcap detectors.

The silicon tracker measures charged particles within the pseudorapidity range $|\eta|<$ 2.5. It consists of silicon pixel and silicon strip detector modules. The electromagnetic calorimeter consists of 75848 lead tungstate crystals that provide coverage in pseudorapidity $|\eta|<1.48$ in a barrel region and $1.48<|\eta|<3.00$ in two endcap regions. The ECAL energy resolution for electrons with a transverse momentum $p_{\mathrm{T}} \approx 45 \mathrm{GeV}$ from $\mathrm{Z} \rightarrow$ ee decays is better than $2 \%$ in the central region of the ECAL barrel $(|\eta|<0.8)$, and is between 2 and $5 \%$ elsewhere [25]. For high energies, which are relevant for this analysis, the electron energy resolution slightly improves [26]. 
Muons are measured in gas-ionization detectors embedded in the steel flux-return yoke outside the solenoid, in the pseudorapidity range $|\eta|<2.4$. Detection is provided using three technologies: drift tubes (DTs), cathode strip chambers (CSCs), and resistive-plate chambers (RPCs). While the barrel region of $|\eta| \leq 1.1$ is instrumented with DTs and RPCs, the endcaps $(1.1<|\eta|<2.4)$ are equipped with CSCs and RPCs. A muon from the interaction point will cross four layers of muon chambers, interleaved with steel forming the return yoke of the magnetic field. Every chamber provides reconstructed hits on several detection planes, which are then combined into local track segments, forming the basis of muon reconstruction inside the muon system. Matching muons to tracks measured in the silicon tracker results in a relative transverse momentum resolution, for muons with $p_{\mathrm{T}}$ up to $100 \mathrm{GeV}$, of $1 \%$ in the barrel and $3 \%$ in the endcaps. The $p_{\mathrm{T}}$ resolution in the barrel is better than $7 \%$ for muons with $p_{\mathrm{T}}$ up to $1 \mathrm{TeV}[27]$.

A particle-flow algorithm [28] aims to reconstruct and identify each individual particle in an event, with an optimized combination of information from the various elements of the CMS detector. The energy of photons is obtained from the ECAL measurement. The energy of electrons is determined from a combination of the electron momentum at the primary interaction vertex as determined by the tracker, the energy of the corresponding ECAL cluster, and the energy sum of all bremsstrahlung photons spatially compatible with originating from the electron track. The muon $p_{\mathrm{T}}$ is obtained from the curvature of the corresponding track. The energy of charged hadrons is determined from a combination of their momentum measured in the tracker and the matching ECAL and HCAL energy deposits, corrected for zero-suppression effects and for the response function of the calorimeters to hadronic showers. Finally, the energy of neutral hadrons is obtained from the corresponding corrected ECAL and HCAL energies.

The CMS experiment has a two-level trigger system [29]. The level-1 trigger, composed of custom hardware processors, selects events of interest using information from the calorimeters and muon detectors and reduces the readout rate from the $40 \mathrm{MHz}$ bunchcrossing frequency to a maximum of $100 \mathrm{kHz}$. The software based high-level trigger uses the full event information, including that from the inner tracker, to reduce the event rate to the $1 \mathrm{kHz}$ that is recorded.

A more detailed description of the CMS detector can be found in ref. [30].

\section{Model and signal simulation}

Excited leptons can be produced at a pp collider by a CI that is described by an effective four-fermion Lagrangian

$$
\mathcal{L}_{\mathrm{CI}}=\frac{g_{*}^{2}}{\Lambda^{2}} \frac{1}{2} j^{\mu} j_{\mu}
$$

with the coupling $g_{*}^{2}$ chosen to correspond to $4 \pi$, fermion current $j_{\mu}=\bar{\psi} \gamma_{\mu} \psi$, and $\Lambda$ representing the compositeness scale [6]. The excited lepton, $\ell^{*}$, can decay to an SM lepton via a CI $\ell^{*} \rightarrow \ell \psi \bar{\psi}$, where $\psi$ is a fermion, or through the mediation of a gauge boson. This analysis complements other searches for excited leptons in the $\ell \ell \gamma$ or $\ell \ell Z$ channels and is 

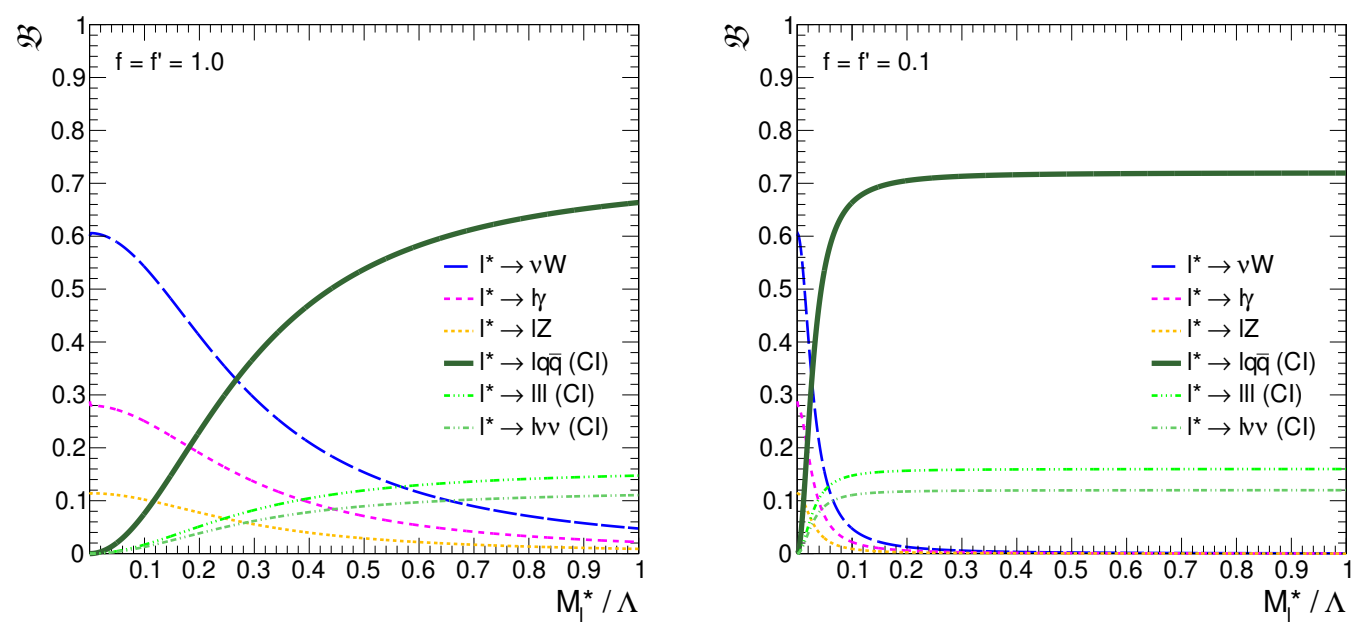

Figure 2. Branching fractions, $\mathcal{B}$, of excited lepton decay channels as a function of the ratio of the excited lepton mass $\left(M_{\ell^{*}}\right)$ and compositeness scale $(\Lambda)$ for fixed values of the model parameters $f=f^{\prime}$, which represent the couplings of excited leptons to SM particles. The branching fraction calculation is based on ref. [6]. The contact interaction decay to one lepton and two jets, subject of this analysis, is dominating the region of high $M_{\ell^{*}} / \Lambda$. Couplings $f$ and $f^{\prime}$ are assumed to be equal to 1 in the left graph, and 0.1 in the right graph.

most sensitive at large values of $M_{\ell^{*}}$ and/or $\Lambda$. Figure 2 illustrates the rapid increase of the branching fraction of the CI decay to a lepton plus two jets as a function of $M_{\ell^{*}} / \Lambda$.

The GI transition of excited leptons to SM leptons is described by

$$
\mathcal{L}_{\mathrm{GI}}=\frac{1}{2 \Lambda} \bar{\psi}_{R}^{\star} \sigma^{\mu \nu}\left(g f \frac{\boldsymbol{\tau}}{2} \boldsymbol{W}_{\mu \nu}+g^{\prime} f^{\prime} \frac{Y}{2} B_{\mu \nu}\right) \psi_{L}+\text { h.c. }
$$

where $g$ and $g^{\prime}$ denote the couplings to the hypercharge and the weak isospin current and $f$ and $f^{\prime}$ effectively scale the energy scale of the interaction $\Lambda$ with respect to the CI [6]. Other quantities are $\boldsymbol{W}_{\mu \nu}$ and $B_{\mu \nu}$ denoting the field strength tensors of the SU(2) and $\mathrm{U}(1)$ gauge fields with their generators $\boldsymbol{\tau}$ and $Y$. The partial width of the CI decay is independent of $f$ and $f^{\prime}$. Interference between GI and CI transitions is not considered [6]. Since the exact values for $f$ and $f^{\prime}$ are not known, searches for excited leptons are typically interpreted for two extreme values: $f=f^{\prime}=1$ and $f=-f^{\prime}=1$. Gauge-interaction decays via photon emission are forbidden for $f=-f^{\prime}$. Figure 2 shows the variation of the branching fractions of the different decay channels as a function of $M_{\ell^{*}} / \Lambda$, illustrated in figure 2 (left) for couplings of 1.0. Couplings below unity are also possible and can have a significant impact on the branching fractions, as seen in figure 2 (right) for couplings of 0.1. Weaker couplings reduce widths for the gauge-interaction decays while enhancing the CI decays. A range of couplings is included in the interpretation of the experimental results.

The production and decay of excited leptons are simulated at leading-order precision with PYTHIA 8.212 [31] using the NNPDF2.3 [32] (in 2016) and NNPDF3.1 [33] (in 2017) parton distribution functions (PDF) and tunes CUETP8M1 (2016) and CP5 (2017) for the underlying event, respectively [34]. Simulated samples are generated for $\ell^{*}$ masses of 200, 500, 750 and $1000 \mathrm{GeV}$ and from 1 to $7 \mathrm{TeV}$ in steps of $0.5 \mathrm{TeV}$. In the simulation, all 
couplings are set to unity and $\Lambda$ to $10 \mathrm{TeV}$. The specific choice of $\Lambda$ in the simulation scales the overall cross section for the process, while leaving the kinematic variables unaffected. Hence, the simulated events can be reweighted in order to represent different choices of the couplings and $\Lambda$. While the resonance width depends on $\Lambda$, it is smaller than the detector resolution for all values used in this analysis. For the example of a $5 \mathrm{TeV}$ signal, the width is of order $15 \%$ of the resolution.

At $\Lambda=10 \mathrm{TeV}$ and $f=1$ the product of the cross section and branching fraction for the channel under study ranges from $0.224 \mathrm{fb}\left(M_{\ell^{*}}=200 \mathrm{GeV}\right), 1.115 \mathrm{fb}\left(M_{\ell^{*}}=1 \mathrm{TeV}\right)$, $1.07 \times 10^{-2} \mathrm{fb}\left(M_{\ell^{*}}=5 \mathrm{TeV}\right)$ to $6.91 \times 10^{-4} \mathrm{fb}\left(M_{\ell^{*}}=7 \mathrm{TeV}\right)$. For a high-mass $\ell^{*}$ signal, each of the decay products will have a $p_{\mathrm{T}}$ of approximately $1 \mathrm{TeV}$, and will tend to be in the central part of the detector.

\section{Event reconstruction}

The candidate vertex with the largest value of summed physics-object $p_{\mathrm{T}}^{2}$ is taken to be the primary pp interaction vertex. Here the physics objects are the jets, clustered using the jet finding algorithm $[35,36]$ with the tracks assigned to the candidate vertices as inputs, and the associated missing transverse momentum, taken as the negative vector $p_{\mathrm{T}}$ sum of these jets. In order to suppress background from cosmic ray muons, an event must have at least one primary vertex, with at least five associated well-reconstructed tracks that are within $24 \mathrm{~cm}$ in the longitudinal and $2 \mathrm{~cm}$ in the transverse direction from the nominal interaction point.

Electrons are reconstructed as ECAL clusters that are matched to a central track and their identification has been optimized for high- $p_{\mathrm{T}}$ values [37]. In order to differentiate between electrons and photons, the properties of the track matched to the calorimeter measurement must be consistent with those of an electron originating from the primary vertex. Specifically, there must be $\leq 1$ hit missing in the innermost tracker layers, and the transverse distance to the primary vertex must be less than $2 \mathrm{~mm}$ (barrel) or less than $5 \mathrm{~mm}$ (endcap). Electron candidates are required to have an electron-like shower shape, and to be within the acceptance region of the barrel $(|\eta|<1.44)$ or the endcaps $(1.56<|\eta|<2.50)$, thus avoiding the transition region between the barrel and the endcap parts of the ECAL. The corresponding track matched to the ECAL cluster must be consistent with a particle originating from the nominal interaction point. The associated energy in the HCAL around the electron direction must be less than $5 \%$ of the reconstructed energy of the electron, once noise and pileup are taken into account. To ensure isolation, the scalar sum of the $p_{\mathrm{T}}$ of all tracker and calorimeter objects in a cone of radius $\Delta R=\sqrt{(\Delta \phi)^{2}+(\Delta \eta)^{2}}<0.3$ where $\phi$ is azimuthal angle in radians, must be less than $3 \%$ of the electron $p_{\mathrm{T}}$. Only wellmeasured tracks that are consistent with originating from the same vertex as the electron are included in the isolation cone.

The muon system covers the pseudorapidity region $|\eta|<2.4$. The reconstruction of muons is optimized for high- $p_{\mathrm{T}}$ values [37]. Measurements from the inner tracker and the outer muon system are combined. The tracker track must have a transverse impact parameter $d_{x y}<2 \mathrm{~mm}$ with respect to the primary vertex. The longitudinal distance of 
the tracker track from the primary vertex must be less than $5 \mathrm{~mm}$. Each muon track is required to have at least one hit in the pixel detector, at least six tracker layer hits, and segments with hits in two or more muon detector stations. Since segments are typically in consecutive layers separated by thick layers of steel, the latter requirement significantly reduces the amount of hadronic punch-through [38]. In order to suppress muons with mismeasured $p_{\mathrm{T}}$, an additional requirement $\sigma_{p_{\mathrm{T}}} / p_{\mathrm{T}}<0.3$ is applied, where $\sigma_{p_{\mathrm{T}}}$ is the $p_{\mathrm{T}}$ uncertainty from the muon track reconstruction. Muon isolation requires that the scalar $p_{\mathrm{T}}$ sum of all tracks originating from the interaction vertex within a $\Delta R<0.3$ cone around its direction, excluding the muon itself, is less than $10 \%$ of the muon's $p_{\mathrm{T}}$.

This analysis uses $\eta$ - and $p_{\mathrm{T}}$-dependent scale factors to correct the simulated event yields for the measured differences in the trigger, reconstruction, and identification efficiencies as compared with data.

For each event, hadronic jets are clustered from the reconstructed particle-flow objects with the infrared and collinear safe anti- $k_{\mathrm{T}}$ jet clustering algorithm $[35,36]$ using a distance parameter of $R=0.4$. Jet momentum is determined as the vectorial sum of all particle momenta in the jet, and is found from simulation to be, on average, within 5 to $10 \%$ of the true momentum over the whole $p_{\mathrm{T}}$ spectrum and detector acceptance. Additional protonproton interactions within the same or nearby bunch crossings can contribute additional tracks and calorimetric energy depositions, increasing the apparent jet momentum. To mitigate this effect, tracks identified as originating from pileup vertices (contributions from additional pp interactions in the same or nearby bunch crossings) are discarded. Jet energy corrections are derived from simulation studies so that the average measured response of jets becomes identical to that of particle level jets. In situ measurements of the momentum balance in dijet, photon + jet, $\mathrm{Z}+$ jet, and multijet events are used to determine any residual differences between the jet energy scale in data and in simulation, and appropriate corrections are made [39]. Additional selection criteria are applied to each jet to remove jets potentially dominated by instrumental effects or reconstruction failures.

\section{Backgrounds}

Because the final state contains two opposite-sign leptons, the main background originates from Drell-Yan (DY) production. These events are simulated using the NLO generator MADGRAPH5_aMC@NLO v2.2.2 [40, 41] with up to two final-state partons, and are hadronized with PYTHIA 8.212. Because large mass lepton pairs associated with large hadronic activity are a small fraction of all DY events, additional high mass simulations are used to ensure sufficient statistics to evaluate this background. The cross section is normalized to next-to-next-to-leading-order (NNLO) QCD and next-to-leading-order (NLO) EWK using a di-lepton mass-dependent $\mathrm{K}$ factor [37, 42].

Another important background is the $t \bar{t}$ process, which yields two prompt leptons if both top quarks decay semi-leptonically. This becomes the dominant background in the medium-mass validation region (VR), as will be described in section 6 . It is simulated with POWHEG 2.0 [43-47] in combination with PYTHIA 8.212 for hadronization. Dedicated highmass $t \bar{t}$ samples are used to provide sufficient number of events to estimate the background 
contribution to the high-mass signal region, and are simulated with the same method as used for the main $t \bar{t}$ sample. The cross section is normalized to NNLO [48]. Production of single top quarks in association with a $\mathrm{W}$-boson or in the $t$-channel production are simulated with Powheg. The single top s-channel is simulated with MADGraph5_amC@NLO.

Several multi-boson processes can also yield at least two leptons. However, because they have small cross sections, they are sub-dominant backgrounds in this search. The $\mathrm{WW}$, WZ, and ZZ processes with at least two leptons are simulated with POWHEG and MadGraph5_amC@NLO. Triple-boson processes are neglected because of their small cross sections.

The $\mathrm{W}+$ jets production can contribute to the signal region when jets are misidentified as electrons. In contrast, the misidentification of muons is negligible. The $\mathrm{W}+\mathrm{jets}$ production is simulated to leading order with MADGRAPH5_aMC@NLO v2.2.2 [40, 41]. In addition, high- $H_{\mathrm{T}} \mathrm{W}$ samples are also used, where $H_{\mathrm{T}}$ is defined as the sum of jet transverse momenta. The $\mathrm{W}+$ jets cross section is normalized to NNLO by application of a K-factor of 1.21 [42].

The background samples are simulated using the NNPDF2.3 or NNPDF3.0 [49] PDF sets with tune CUETP8M1 (in 2016) and the NNPDF3.1 (in 2017) PDF set with CP5 (2017) for the underlying event, respectively [34]. All generated events are processed through a full simulation of the CMS detector based on GEANT4 [50], a trigger emulation, and the event reconstruction chain. All simulated event samples are normalized to the integrated luminosity of the recorded data. The simulation of pileup is included in all events by superimposing simulated minimum bias interactions. For the data set used, the average number of interactions per bunch crossing after selection is about 23 in 2016 and about 30 in 2017, with maximum values of 55 and 70 , respectively.

\section{$6 \quad$ Event selection and validation regions}

Events with electrons in the final state were selected using a trigger that requires an electromagnetic cluster (electron or photon) with transverse momenta larger than $175 \mathrm{GeV}$ (for 2016) and $200 \mathrm{GeV}$ (for 2017). Events with muons in the final state were triggered with the single-muon trigger with a minimum $p_{\mathrm{T}}$ of $50 \mathrm{GeV}$. The electron (muon) efficiency plateaus at $98.5 \%(98 \%)[51]$ as a function of $p_{\mathrm{T}}$ above a turn-on region and is independent of $\eta, \phi$, and pileup.

Final state particles (electrons, muons and jets) are reconstructed as described in section 4 . The two highest $p_{\mathrm{T}}$ (leading) leptons are selected along with the two leading jets. The $p_{\mathrm{T}}$ thresholds for the leading lepton are $230 \mathrm{GeV}$ (electron) and $53 \mathrm{GeV}$ (muon), chosen to be above the respective trigger turn-on regions. The subleading electrons or muons are required to have an offline $p_{\mathrm{T}}$ of at least 35 and $25 \mathrm{GeV}$, respectively. Jets are required to have a minimum $p_{\mathrm{T}}$ of $50 \mathrm{GeV}$. Events with more than two same-flavor leptons are rejected, to reduce background from diboson production.

The majority of the DY background is suppressed by requiring $M_{\ell \ell}>500 \mathrm{GeV}$, constituting the high-mass signal region (SRT). Two VRs are then defined, illustrated in figure 3, to verify agreement between data and simulation in different mass regions. The low-mass 


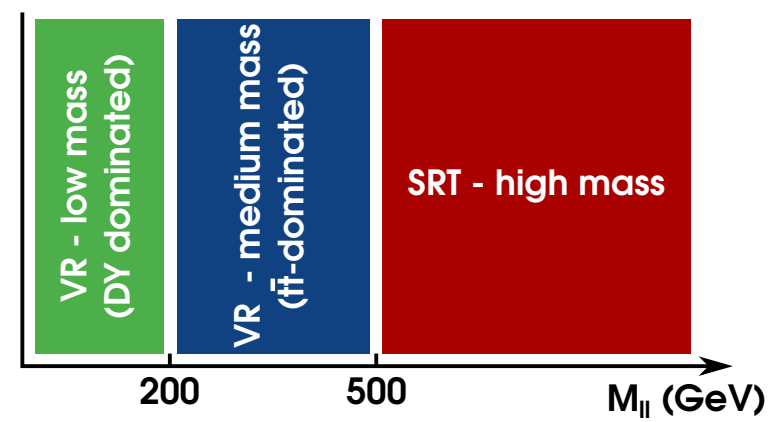

Figure 3. Definition of the two validation regions (VR) and the high-mass signal region (SRT).
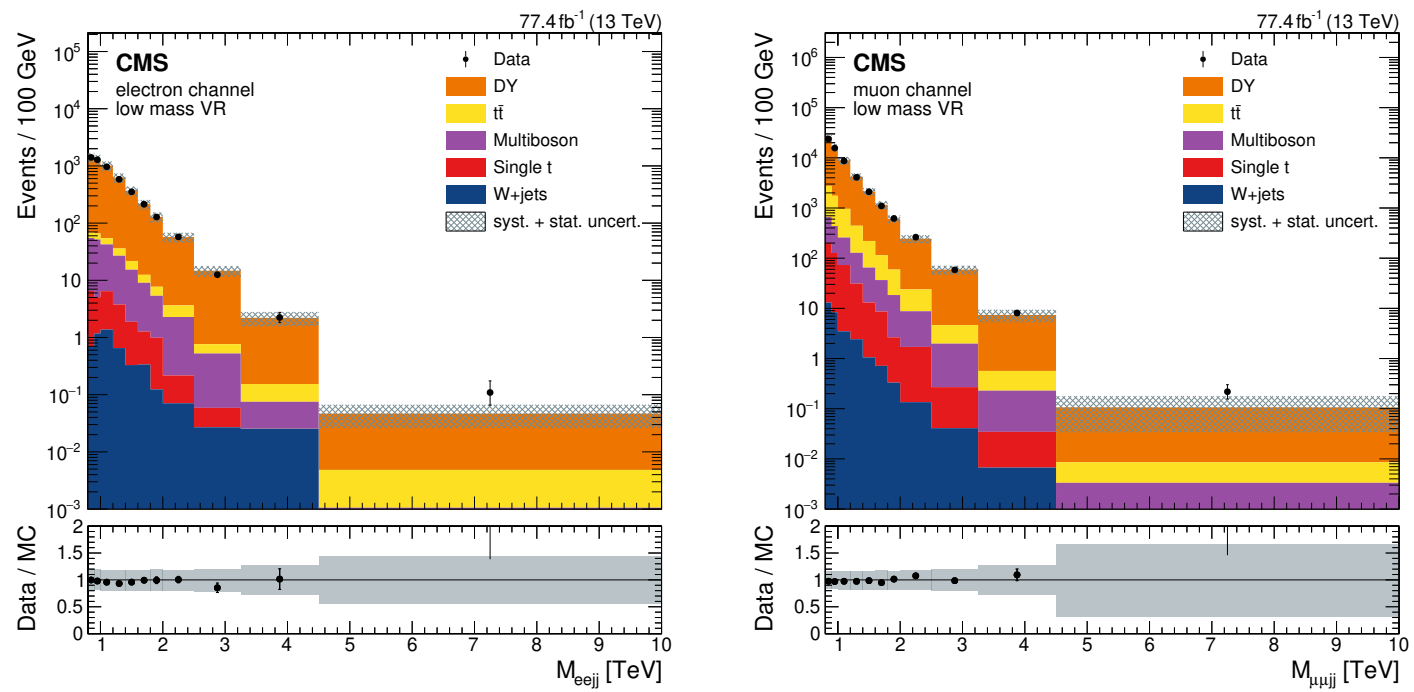

Figure 4. Event distributions as a function of the four-body invariant $M_{\ell \ell j j}$ mass for the electron (left) and muon (right) channels, for the low-mass validation region defined by $M_{\ell \ell}<200 \mathrm{GeV}$. The lower panels show the ratio of data to the simulated SM background, with the shaded band representing the uncertainty.

$V R$ with $M_{\ell \ell}<200 \mathrm{GeV}$ serves as the VR for DY, which is the dominant background for this final state. The subleading t $\overline{\mathrm{t}}$ background tends to populate the medium-mass $V R$, which is defined by $200 \mathrm{GeV}<M_{\ell \ell}<500 \mathrm{GeV}$. While the low-mass VR is nearly signal free, the medium-mass VR potentially contains a very small fraction of signal events. The signal contamination in the medium VR is far below $1 \%$ for the interesting mass range of $M_{\ell^{*}}$ above $3.8 \mathrm{TeV}$.

The invariant mass of the combination of both selected leptons and the two leading jets, $M_{\ell \ell j j}$, is used as the discriminating variable as it provides the best separation power between signal and background. The distributions of other possible variables were studied, but their use yielded lower signal significance. The event distributions as functions of the four-body mass for the low-mass and medium-mass validation regions are shown in figures 4 and 5 , respectively. The good agreement between experimental and simulated data justifies estimating the background expectation from simulation. 

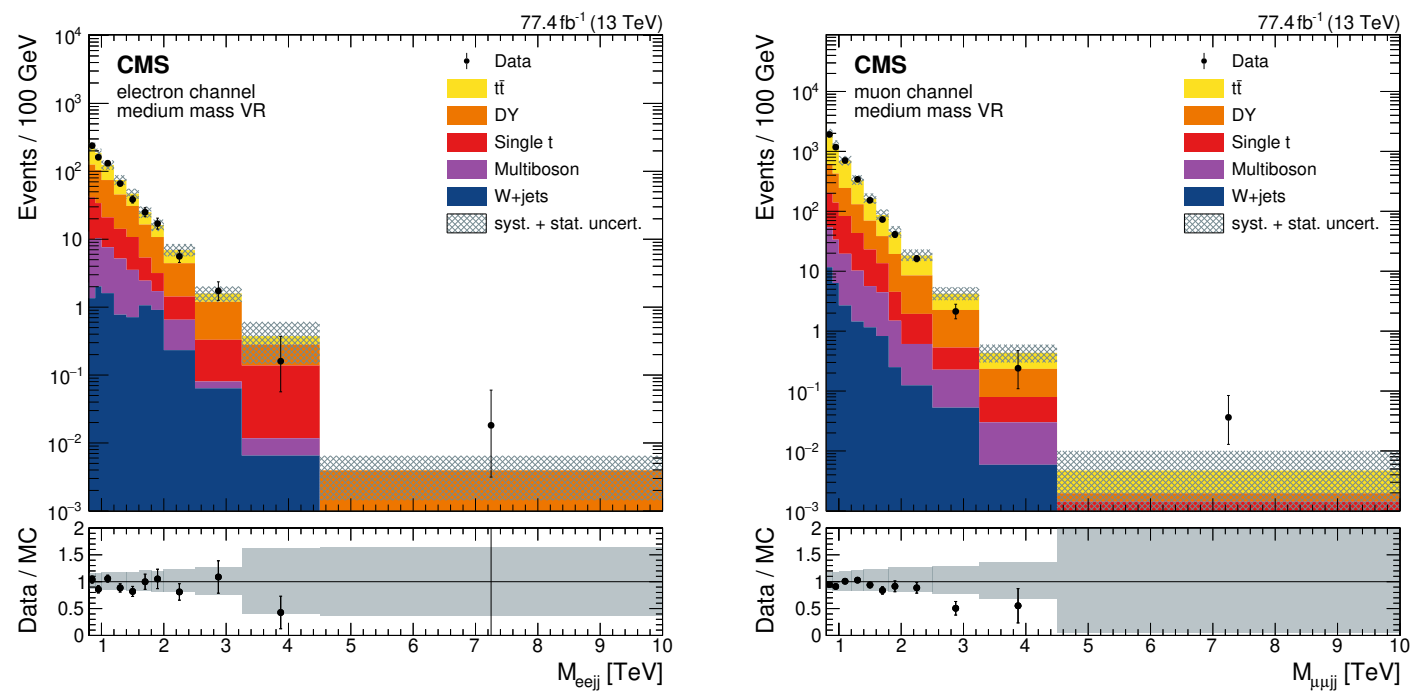

Figure 5. Event distribution as a function of the four-body invariant mass $M_{\ell \ell j j}$ for the electron (left) and muon (right) channels, for the medium-mass validation region defined by $200<M_{\ell \ell}<$ $500 \mathrm{GeV}$. The lower panel shows the ratio of data to the simulated SM background, with the shaded band representing the uncertainty.

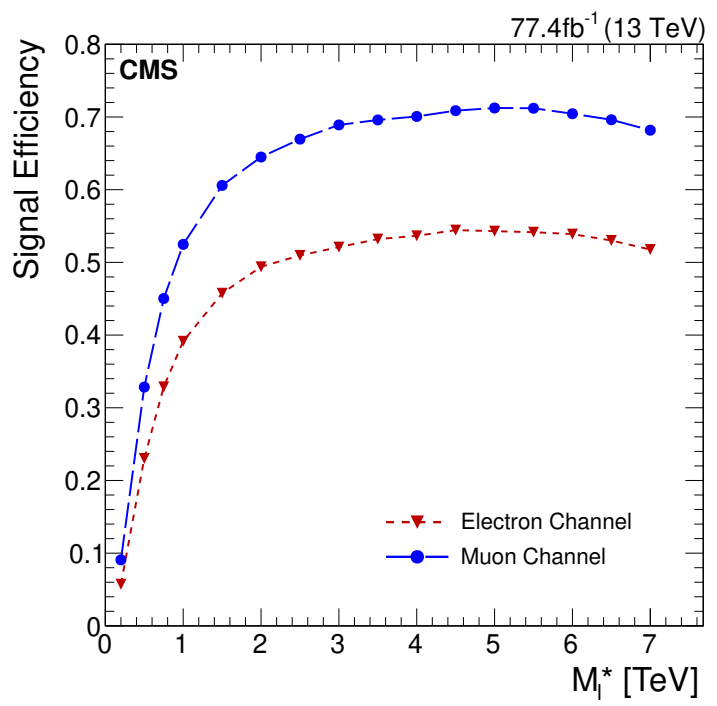

Figure 6. Signal efficiency after all selections are applied, as a function of the excited lepton mass $M_{\ell^{*}}$, based on simulated events.

The signal efficiency, defined as the product of acceptance and detection efficiency of the fraction of reconstructed $2 \ell 2 \mathrm{j}$ events, resulting from these event selections is shown as a function of the simulated excited lepton mass in figure 6 . The uncertainties in the figure are only statistical uncertainties. The systematic uncertainties on the product of acceptance and efficiency are between 6 and $8 \%$. The efficiency reaches a plateau of about $55 \%$ for the electron channel and $73 \%$ for the muon channel. For lower masses, in particular for $M_{\ell^{*}}<1 \mathrm{TeV}$, the acceptance is reduced by the offline $p_{\mathrm{T}}$ thresholds on the objects but 
this mass region is not of primary interest given previous exclusion limits. For masses above $6 \mathrm{TeV}$, the efficiency starts to drop slightly because of a growing fraction of events produced off-shell. At the given center-of-mass energy of the LHC, the available energy for generating such heavy particles is limited.

\section{Systematic uncertainties}

Systematic uncertainties in this analysis can affect the overall normalization and also the shape of the distributions. Uncertainties in the energy scale of different physics objects are taken into account by analyzing the shape of the four-body mass distributions, with the energy scale shifted up and down by $1 \sigma$. The electron energy scale uncertainty is estimated to be $0.2 \%$ in the barrel and $0.3 \%$ in the endcap [52]. For muons, the momentum scale is determined using the generalized endpoint method [27] and applied as a function of the $\eta$ and $\phi$ of the muon. The studies indicate the absence of a significant curvature bias, within an uncertainty of $0.02 / \mathrm{TeV}$ in the central region and up to $0.1 / \mathrm{TeV}$ in the more forward regions. For jets, the uncertainties associated with the jet energy correction are used [39].

The simulated energy resolution is better than that measured in data. To account for this, jet energy resolution corrections are applied to jets and their uncertainties are considered as a systematic effect [39]. For electrons, the momentum is smeared by 1.2 and $2.4 \%$ for barrel and endcaps, respectively [52]. For muons, the momentum is smeared by $5 \%$ [27]. These three uncertainties change the shape of the background.

There are systematic uncertainties on the scale factors applied to correct for the differences in the triggering, reconstruction and identification efficiencies between simulation and data. These uncertainties are typically a few percent and are taken into account as normalization uncertainty $[27,52]$. The integrated luminosity of the data collected has an uncertainty of 2.5 [53] and 2.3\% [54] for the 2016 and 2017 data sets, respectively. The uncertainty due to the modeling of pileup is found to be less than $10 \%$ and is by derived by calculating the pileup for the cases where the total inelastic cross section is increased and decreased by $5 \%$ from the nominal value [55]. It is treated as an uncertainty in the background shape.

The uncertainty associated with the choice of PDFs affects the cross section of the simulated samples and is taken into account by following an approach outlined by the PDF4LHC recipe [56]. The PDF set used to calculate the variations is NNPDF3.0, for background and signal samples in 2016, and NNPDF3.1, in 2017. Overall, the variation is found to range from $5 \%$ to $60 \%$ for an excited lepton mass from $1 \mathrm{TeV}$ to $6 \mathrm{TeV}$, respectively, as taken from LHAPDF6.2 [57].

The renormalization and factorization scales provide a handle to estimate the uncertainty due to missing higher orders. Both scales are varied simultaneously by a factor 2 up and down, resulting in different event-by-event weights and an uncertainty of 10 to $30 \%$ between 1 and $6 \mathrm{TeV}$.

In the signal region, the limited number of simulated background events results in a statistical uncertainty of up to $40 \%$ in the background prediction. 

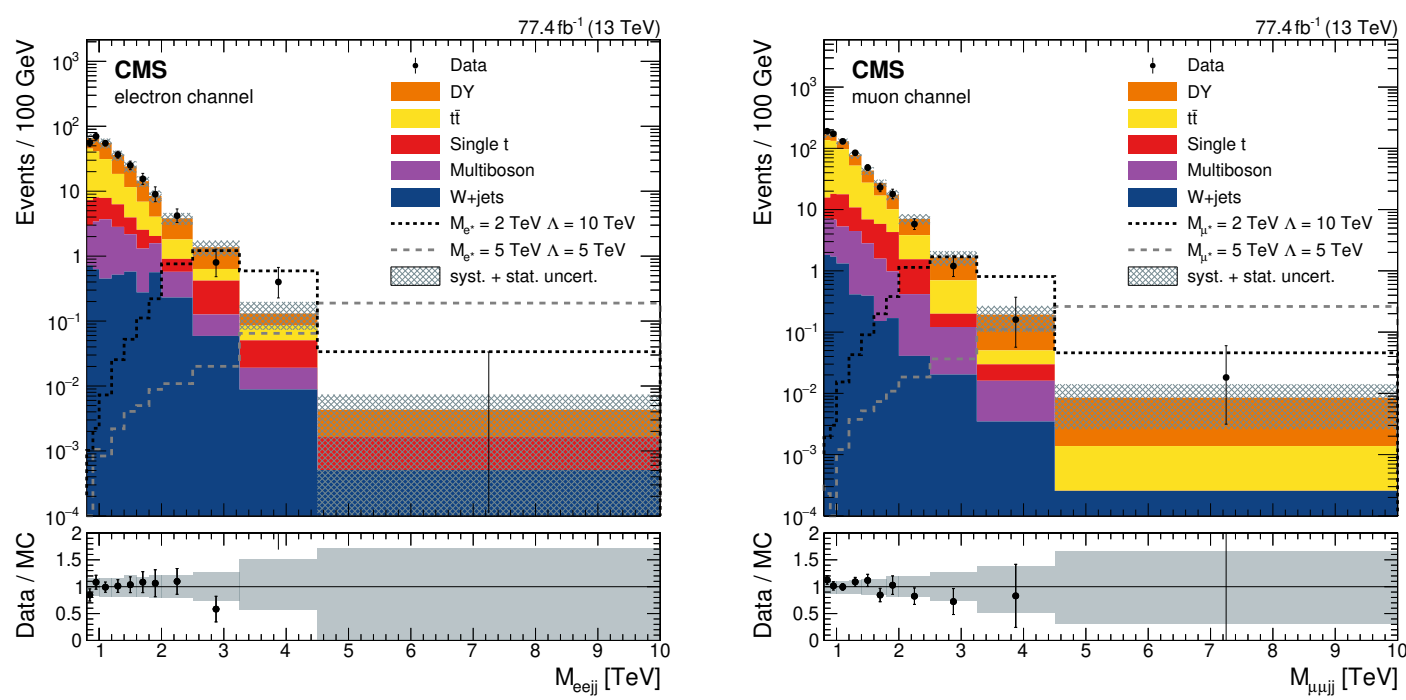

Figure 7. Distribution of the two-lepton two-jet invariant mass in the signal region $\left(M_{\ell \ell}>\right.$ $500 \mathrm{GeV}$ ) for the electron (left) and muon (right) channels. The example signal shape for two excited lepton masses is indicated as a gray line with the parameters given in the legend and for the benchmark case where the couplings $f$ and $f^{\prime}$ are set to unity. The lower panels show the ratio of data to simulation with the total uncertainty in gray.

Production of $\mathrm{W}+$ jets final states can contribute to the background when a jet is misidentified as a lepton, albeit at a very small level. A conservative systematic uncertainty of $100 \%$ is applied to the $\mathrm{W}+$ jets background contribution and reflects the uncertainty in the lepton misidentification rate in the simulation as compared with data.

For the SM background, the dominant sources of uncertainties are the Monte-Carlo (MC) sample sizes and the PDF uncertainty.

\section{Results}

Event distributions for the signal regions as a function of the four-body invariant mass are shown for the electron and muon channels in figure 7. The final distribution is dominated by $t \overline{\mathrm{t}}$ background in the low-mass region and DY background in the high mass region despite being strongly suppressed by the dilepton invariant mass cut. The expected background in the highest mass bin, of more than $5 \mathrm{TeV}$ width, is far less than one event. The analysis would not benefit from reducing the background further at the cost of signal efficiency. The event yields in data compared to the total expected SM backgrounds are given in table 1 for a number of bins of the discriminating four-body $2 \ell 2 j$ mass distribution. Also shown are the expectations for potential $\ell^{*}$ signals with two different sets of model parameter values: a mass $M_{\ell^{*}}$ of $2 \mathrm{TeV}$ and compositeness scale $\Lambda$ of $10 \mathrm{TeV}$; and $M_{\ell^{*}}=\Lambda=5 \mathrm{TeV}$, which roughly corresponds to the maximum sensitivity of this analysis.

No indication of a signal is observed. The limits on the excited lepton mass, $M_{\ell^{*}}$, are calculated using the Bayesian method [58] with a uniform positive prior probability distribution for the signal cross section. Systematic uncertainties in the expected signal and background yields are included either via nuisance parameters with log-normal 


\begin{tabular}{|lccccc|}
\hline \multicolumn{5}{c}{ Bins in $2 \ell 2 j$ mass $(\mathrm{GeV})$} \\
\hline Data in $2 e 2 j$ & $800-1600$ & $1600-2500$ & $2500-3250$ & $3250-4500$ & $4500-10000$ \\
SM prediction & 359 & 70 & 6 & 5 & 0 \\
& $362_{-61}^{+58}$ & $65 \pm 12$ & $10.3_{-2.8}^{+2.7}$ & $1.6_{-0.7}^{+0.8}$ & $0.24_{-0.24}^{+0.17}$ \\
$f=f^{\prime}=1$ & & & & \\
$M_{\mathrm{e}^{*}}=2 \mathrm{TeV}, \Lambda=10 \mathrm{TeV}$ & $0.17_{-0.02}^{+0.01}$ & $4.5 \pm 0.3$ & $9.1 \pm 0.6$ & $7.4 \pm 0.5$ & $1.9 \pm 0.2$ \\
$M_{\mathrm{e}^{*}}=5 \mathrm{TeV}, \Lambda=5 \mathrm{TeV}$ & $0.015_{-0.003}^{+0.002}$ & $0.08 \pm 0.01$ & $0.15 \pm 0.01$ & $0.81_{-0.06}^{+0.07}$ & $10.4 \pm 0.7$ \\
$f=f^{\prime}=0.1$ & & & & \\
$M_{\mathrm{e}^{*}}=2 \mathrm{TeV}, \Lambda=10 \mathrm{TeV}$ & $0.53 \pm 0.05$ & $13.6_{-0.9}^{+1.0}$ & $27.8_{-1.8}^{+1.7}$ & $22.6 \pm 1.6$ & $5.7 \pm 0.5$ \\
$M_{\mathrm{e}^{*}}=5 \mathrm{TeV}, \Lambda=5 \mathrm{TeV}$ & $0.016_{-0.003}^{+0.002}$ & $0.09 \pm 0.01$ & $0.16 \pm 0.01$ & $0.85_{-0.07}^{+0.08}$ & $10.9 \pm 0.8$ \\
\hline Data in $2 \mu 2 j$ & 889 & 111 & 9 & 2 & 1 \\
$\mathrm{SM}$ prediction & $842_{-102}^{+91}$ & $125_{-19}^{+18}$ & $12.4 \pm 3.3$ & $2.4_{-1.2}^{+0.9}$ & $0.47_{-0.32}^{+0.31}$ \\
& & & & \\
$f=f^{\prime}=1$ & & & & & \\
$M_{\mu^{*}}=2 \mathrm{TeV}, \Lambda=10 \mathrm{TeV}$ & $0.30 \pm 0.02$ & $6.9_{-0.2}^{+0.3}$ & $12.7_{-0.4}^{+0.5}$ & $10.1_{-0.4}^{+0.6}$ & $2.5 \pm 0.2$ \\
$M_{\mu^{*}}=5 \mathrm{TeV}, \Lambda=5 \mathrm{TeV}$ & $0.021 \pm 0.003$ & $0.13 \pm 0.01$ & $0.27_{-0.01}^{+0.02}$ & $1.4 \pm 0.1$ & $14.4_{-0.4}^{+1.2}$ \\
$f=f^{\prime}=0.1$ & & & & & \\
$M_{\mu^{*}}=2 \mathrm{TeV}, \Lambda=10 \mathrm{TeV}$ & $0.92_{-0.05}^{+0.06}$ & $20.9_{-0.7}^{+0.9}$ & $38.8_{-1.1}^{+1.6}$ & $30.8_{-1.1}^{+1.8}$ & $7.7_{-0.5}^{+0.7}$ \\
$M_{\mu^{*}}=5 \mathrm{TeV}, \Lambda=5 \mathrm{TeV}$ & $0.021 \pm 0.003$ & $0.14 \pm 0.01$ & $0.29_{-0.01}^{+0.02}$ & $1.5 \pm 0.1$ & $15.1_{-0.4}^{+1.2}$ \\
\hline
\end{tabular}

Table 1. Observed event yields in bins of four-body mass compared to the expected SM background, for the $2 e 2 j$ and $2 \mu 2 j$ final states. Also shown are the expected event yields for two simulated signal samples with the given masses and couplings. All yields are given in bins of the discriminating four-body mass $(2 \ell 2 j)$ distribution, with lower and upper value for each bin given in units of GeV. Systematic uncertainties, as described in the text, are shown.

prior distributions or with the shape of the distribution included through the use of a binned likelihood.

Limits at $95 \%$ confidence level (CL) on the product of cross section and branching fraction assuming the proper branching fraction for $M_{\ell^{*}}=\Lambda$ and $f=f^{\prime}=1$ are shown in figure 8 , and range from 10 to $0.1 \mathrm{fb}$, depending on the mass of the excited lepton.

This analysis excludes excited electrons and excited muons at $95 \%$ CL for values of $M_{\ell^{*}}$ below 5.6 and $5.7 \mathrm{TeV}$, respectively, assuming that $\Lambda$ is equal to the mass of the excited leptons. These are the best limits to date on excited electrons and muons. At low masses, the sensitivity is determined by the acceptance and analysis selection. At very high mass, the sensitivity becomes limited by the cross section. The sensitivity to the maximum $\ell^{*}$ mass is not affected by the coupling strength.

The cross section limit can be re-evaluated in terms of the compositeness scale $\Lambda$ as a function of the excited lepton mass. This sensitivity does depend on the coupling strength. Figures $9-11$ show the variations in the compositeness scale $\Lambda$ for the gauge couplings $|f|$ and $\left|f^{\prime}\right|$ equal to unity, for $|f|$ and $\left|f^{\prime}\right|$ equal to 0.1 , and for $f$ and $f^{\prime}$ equal to zero, respectively. Compositeness scales below $5 \mathrm{TeV}$ are excluded for all investigated $\ell^{*}$ masses. 

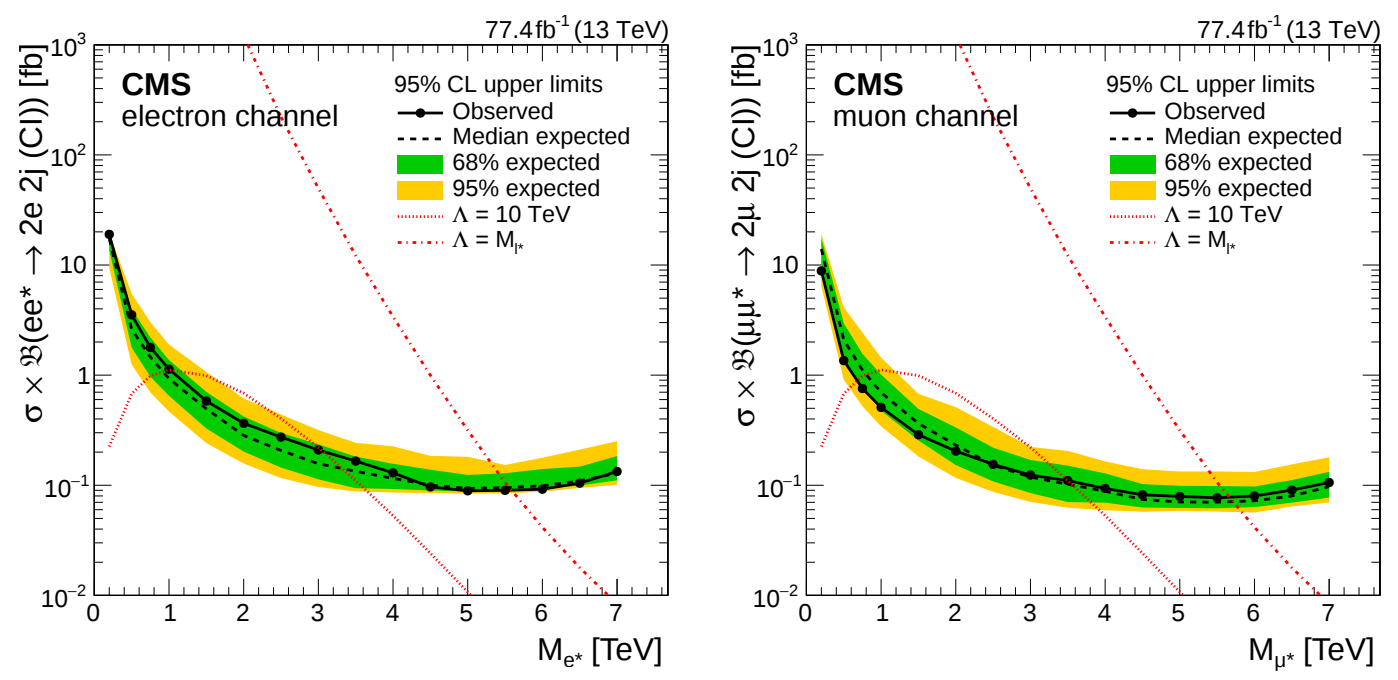

Figure 8. Limits at $95 \% \mathrm{CL}$ on the product of the production cross section and branching fraction for $\ell \ell^{*} \rightarrow \ell \ell j j$, as a function of the excited lepton mass $M_{\ell^{*}}$, for the electron (left) and muon (right) channels. The expectation from the model is represented for $|f|=\left|f^{\prime}\right|=1$ by two cases, $\Lambda=10 \mathrm{TeV}$, and $\Lambda=M_{\ell^{*}}$.
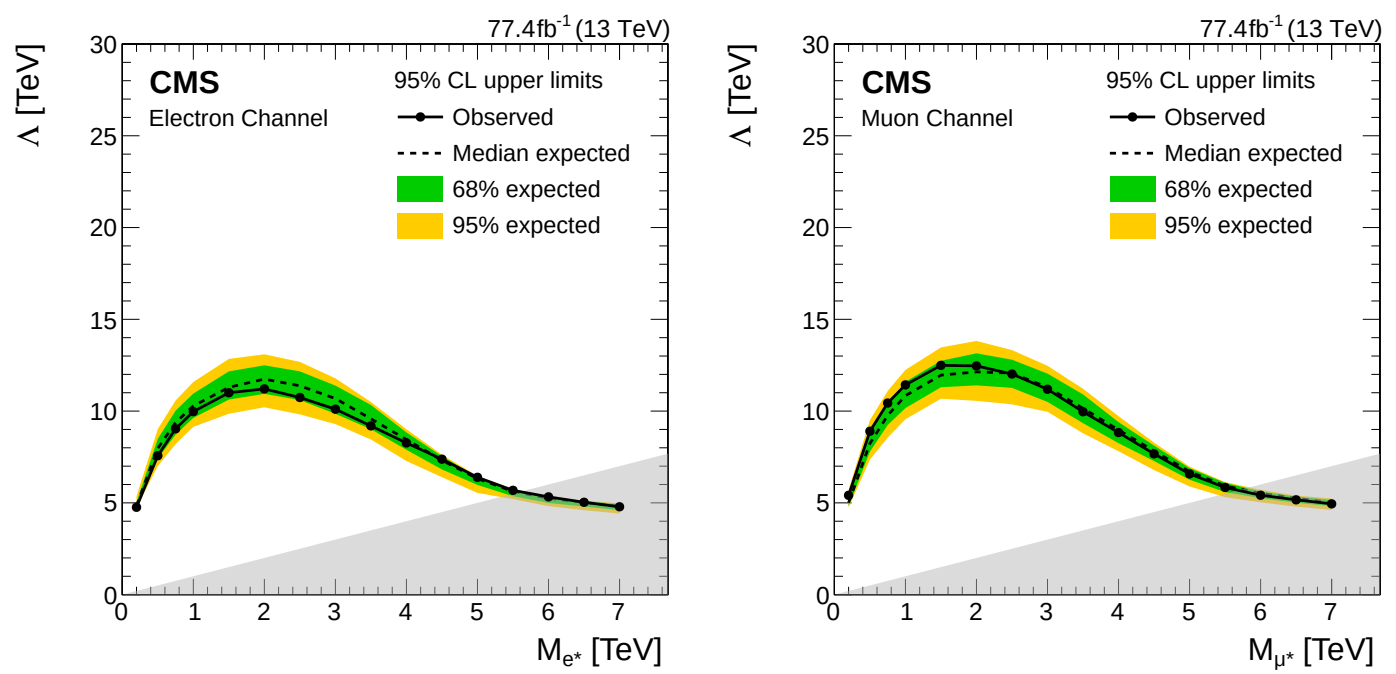

Figure 9. Limits on the compositeness scale $\Lambda$ for the electron (left) and muon (right) channels, as a function of the mass of the excited lepton, for the benchmark case where the GI couplings $|f|$ and $\left|f^{\prime}\right|$ are set to one. The model is not valid in the hatched area.

Maximum sensitivity to $\Lambda$ is reached at low masses, as is typical for excited lepton searches. The exact mass for this peak sensitivity depends on the coupling scenario, decreasing with weaker GI couplings, and can easily be understood from figure 2. Detailed numbers are given in table 2. In the case of $f=f^{\prime}=1$, compositeness scales up to $11 \mathrm{TeV}$ ( $\mathrm{e}^{*}$ ) and $12 \mathrm{TeV}\left(\mu^{*}\right)$ are excluded, with the maximum sensitivity for $\ell^{*}$ masses around $2 \mathrm{TeV}$. With values for $f=f^{\prime}=0.1$, the decay width to a CI increases, yielding a higher $\Lambda$ sensitivity of $17 \mathrm{TeV}\left(\mathrm{e}^{*}\right)$ and $19 \mathrm{TeV}\left(\mu^{*}\right)$, respectively, for $\ell^{*}$ masses around $1.5 \mathrm{TeV}$. Assuming zero GI couplings, the observed $\Lambda$ sensitivity increases to $18 \mathrm{TeV}\left(\mathrm{e}^{*}\right)$ and $22 \mathrm{TeV}\left(\mu^{*}\right)$, 

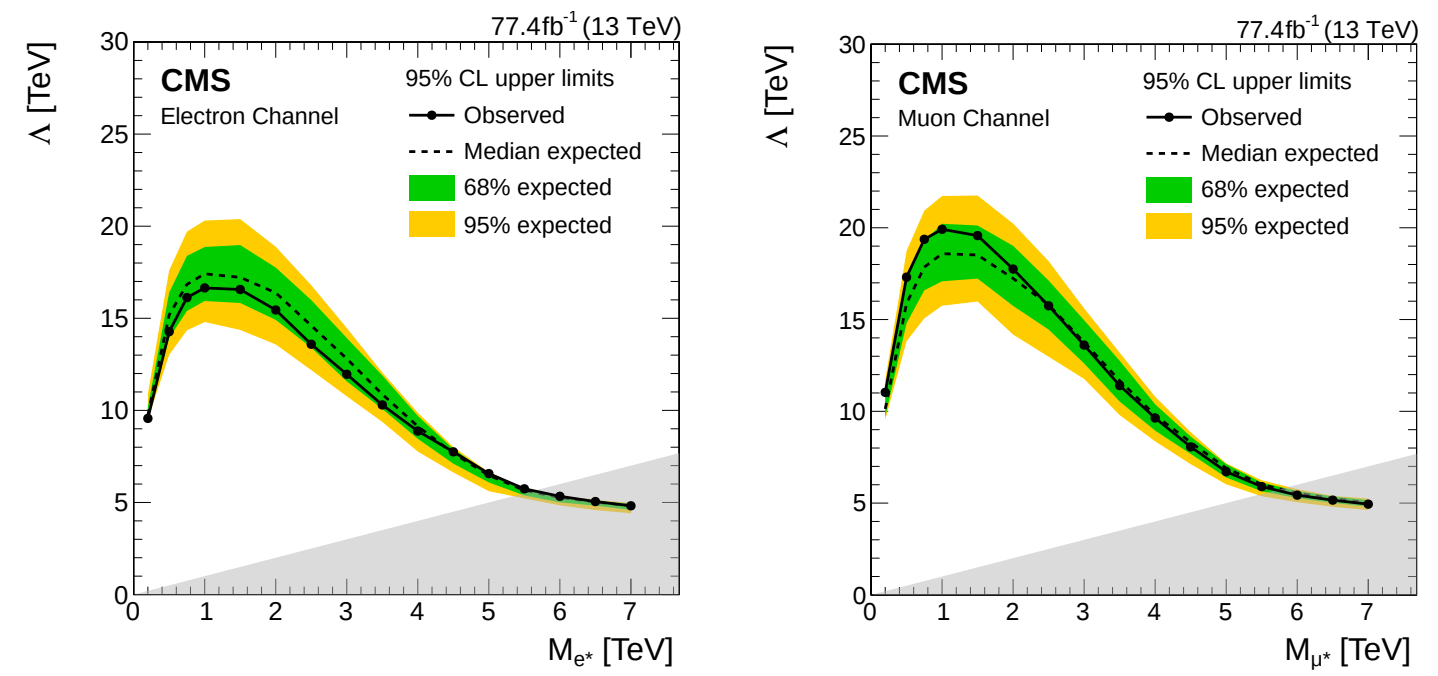

Figure 10. Limits on the compositeness scale $\Lambda$ for the electron (left) and muon (right) channels, as a function of the mass of the excited lepton, for the case where the GI couplings $|f|$ and $\left|f^{\prime}\right|$ are set to 0.1 . The model is not valid in the hatched area.
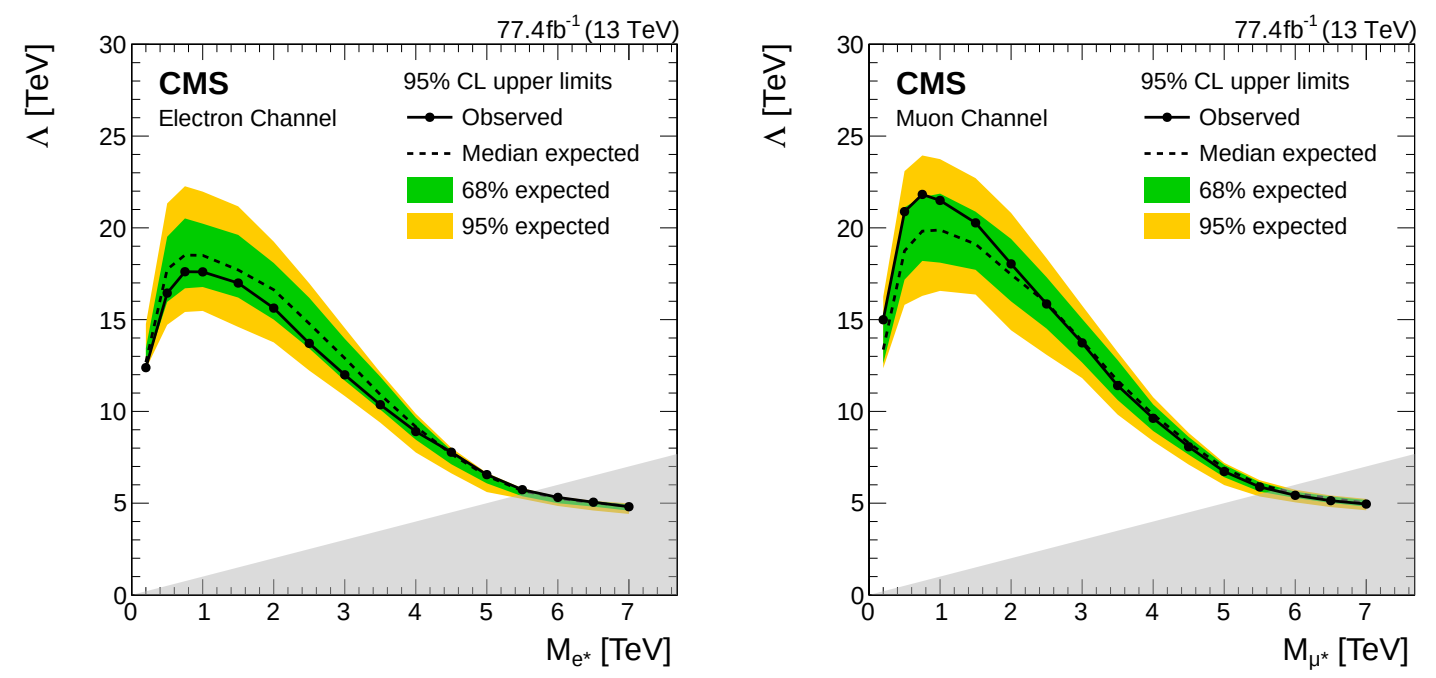

Figure 11. Limits on the compositeness scale $\Lambda$ for the electron (left) and muon (right) channels, as a function of the mass of the excited lepton, for the benchmark case where the GI couplings $f$ and $f^{\prime}$ vanish. The model is not valid in the hatched area.

respectively, for $\ell^{*}$ masses around $1 \mathrm{TeV}$. While the expected sensitivities in the electron and muon channels are comparable, the observed muon channel sensitivity is up to one standard deviation higher due to fluctuations in the data. For $M_{\ell^{*}}$ above $2 \mathrm{TeV}$ these are the best limits to date. In the scenario $f=-f^{\prime}$, where $\ell \ell \gamma$ has no sensitivity, this CI channel provides the best test of high compositeness scales. 


\begin{tabular}{|llccl|}
\hline \multirow{2}{*}{ Search channel } & $\begin{array}{l}\text { Coupling } \\
\text { strength }\end{array}$ & \multicolumn{2}{l|}{$M_{\ell^{*}}=\Lambda$, values in $\mathrm{TeV}$} & Limit on $\Lambda$, in $\mathrm{TeV}$ \\
& $f=f^{\prime}$ & $f=-f^{\prime}$ & \\
\hline $\mathrm{ee}^{*} \rightarrow 2 \mathrm{e} 2 j$ & $f=1$ & $5.6(5.6)$ & $5.6(5.6)$ & $11(11)$ for $M_{\ell^{*}} \approx 2 \mathrm{TeV}$ \\
& $f=0.1$ & $5.6(5.6)$ & $5.6(5.6)$ & $17(18)$ for $M_{\ell^{*}} \approx 1.5 \mathrm{TeV}$ \\
& $f=0$ & $5.6(5.6)$ & $5.6(5.6)$ & $18(19)$ for $M_{\ell^{*}} \approx 1 \mathrm{TeV}$ \\
$\mu \mu^{*} \rightarrow 2 \mu 2 j$ & $f=1$ & $5.7(5.7)$ & $5.7(5.7)$ & $12(12)$ for $M_{\ell^{*}} \approx 2 \mathrm{TeV}$ \\
& $f=0.1$ & $5.7(5.7)$ & $5.7(5.7)$ & $19(19)$ for $M_{\ell^{*}} \approx 1.5 \mathrm{TeV}$ \\
& $f=0$ & $5.7(5.7)$ & $5.7(5.7)$ & $22(20)$ for $M_{\ell^{*}} \approx 1 \mathrm{TeV}$ \\
\hline
\end{tabular}

Table 2. Summary of the observed (expected) limits on $\ell^{*}$ mass, assuming $M_{\ell^{*}}=\Lambda$, for the cases $f=f^{\prime}$ and $f=-f^{\prime}$. The limits evaluated in terms of the compositeness scale $\Lambda$ are shown in the right column.

\section{Summary}

A search for excited leptons decaying via a contact interaction to final states of two electrons or two muons and two resolved jets has been performed. This channel complements other searches for excited leptons. It has greatest sensitivity at large values of the excited lepton mass $M_{\ell^{*}}$. The data for this analysis were recorded with the CMS detector in the years 2016 and 2017, corresponding to a total integrated luminosity of $77.4 \mathrm{fb}^{-1}$ of proton-proton collisions at a center-of-mass energy of $13 \mathrm{TeV}$.

No significant deviations from SM expectations are observed in the signal region and 95\% exclusion limits have been set. Excited electrons (muons) up to masses of $M_{\mathrm{e}^{*}}=$ $5.6 \mathrm{TeV}\left(M_{\mu^{*}}=5.7 \mathrm{TeV}\right)$ are excluded with the usual assumption of $M_{\ell^{*}}=\Lambda$. These are the best limits to date. The limit was also re-evaluated in terms of the substructure scale $\Lambda$, leading to limits of $\Lambda=11$ and $12 \mathrm{TeV}$ for excited electrons and muons, respectively, for mass values around $2 \mathrm{TeV}$ and couplings of unity. When studying weaker gauge couplings, the limit on the maximum $M_{\ell^{*}}$ does not change, but the larger cross section increases the $\Lambda$ sensitivity at lower masses. For couplings around zero, where the $\ell \ell \gamma$ decay has no sensitivity, limits around $20 \mathrm{TeV}$ for the compositeness scale $\Lambda$ are achieved.

\section{Acknowledgments}

We congratulate our colleagues in the CERN accelerator departments for the excellent performance of the LHC and thank the technical and administrative staffs at CERN and at other CMS institutes for their contributions to the success of the CMS effort. In addition, we gratefully acknowledge the computing centers and personnel of the Worldwide LHC Computing Grid for delivering so effectively the computing infrastructure essential to our analyses. Finally, we acknowledge the enduring support for the construction and operation of the LHC and the CMS detector provided by the following funding agencies: BMBWF and FWF (Austria); FNRS and FWO (Belgium); CNPq, CAPES, FAPERJ, FAPERGS, and FAPESP (Brazil); MES (Bulgaria); CERN; CAS, MoST, and NSFC (China); COLCIENCIAS (Colombia); MSES and CSF (Croatia); RPF (Cyprus); SENESCYT (Ecuador); 
MoER, ERC IUT, PUT and ERDF (Estonia); Academy of Finland, MEC, and HIP (Finland); CEA and CNRS/IN2P3 (France); BMBF, DFG, and HGF (Germany); GSRT (Greece); NKFIA (Hungary); DAE and DST (India); IPM (Iran); SFI (Ireland); INFN (Italy); MSIP and NRF (Republic of Korea); MES (Latvia); LAS (Lithuania); MOE and UM (Malaysia); BUAP, CINVESTAV, CONACYT, LNS, SEP, and UASLP-FAI (Mexico); MOS (Montenegro); MBIE (New Zealand); PAEC (Pakistan); MSHE and NSC (Poland); FCT (Portugal); JINR (Dubna); MON, RosAtom, RAS, RFBR, and NRC KI (Russia); MESTD (Serbia); SEIDI, CPAN, PCTI, and FEDER (Spain); MOSTR (Sri Lanka); Swiss Funding Agencies (Switzerland); MST (Taipei); ThEPCenter, IPST, STAR, and NSTDA (Thailand); TUBITAK and TAEK (Turkey); NASU (Ukraine); STFC (United Kingdom); DOE and NSF (U.S.A.).

Individuals have received support from the Marie-Curie program and the European Research Council and Horizon 2020 Grant, contract Nos. 675440, 752730, and 765710 (European Union); the Leventis Foundation; the A.P. Sloan Foundation; the Alexander von Humboldt Foundation; the Belgian Federal Science Policy Office; the Fonds pour la Formation à la Recherche dans l'Industrie et dans l'Agriculture (FRIA-Belgium); the Agentschap voor Innovatie door Wetenschap en Technologie (IWT-Belgium); the F.R.S.-FNRS and FWO (Belgium) under the "Excellence of Science - EOS" — be.h project n. 30820817; the Beijing Municipal Science \& Technology Commission, No. Z191100007219010; the Ministry of Education, Youth and Sports (MEYS) of the Czech Republic; the Deutsche Forschungsgemeinschaft (DFG) under Germany's Excellence Strategy — EXC 2121 "Quantum Universe" - 390833306; the Lendület ("Momentum") Program and the János Bolyai Research Scholarship of the Hungarian Academy of Sciences, the New National Excellence Program ÚNKP, the NKFIA research grants 123842, 123959, 124845, 124850, 125105, 128713, 128786, and 129058 (Hungary); the Council of Science and Industrial Research, India; the HOMING PLUS program of the Foundation for Polish Science, cofinanced from European Union, Regional Development Fund, the Mobility Plus program of the Ministry of Science and Higher Education, the National Science Center (Poland), contracts Harmonia 2014/14/M/ST2/00428, Opus 2014/13/B/ST2/02543, 2014/15/B/ST2/03998, and 2015/19/B/ST2/02861, Sonata-bis 2012/07/E/ST2/01406; the National Priorities Research Program by Qatar National Research Fund; the Ministry of Science and Education, grant no. 14.W03.31.0026 (Russia); the Programa Estatal de Fomento de la Investigación Científica y Técnica de Excelencia María de Maeztu, grant MDM-2015-0509 and the Programa Severo Ochoa del Principado de Asturias; the Thalis and Aristeia programs cofinanced by EU-ESF and the Greek NSRF; the Rachadapisek Sompot Fund for Postdoctoral Fellowship, Chulalongkorn University and the Chulalongkorn Academic into Its 2nd Century Project Advancement Project (Thailand); the Kavli Foundation; the Nvidia Corporation; the SuperMicro Corporation; the Welch Foundation, contract C-1845; and the Weston Havens Foundation (U.S.A.).

Open Access. This article is distributed under the terms of the Creative Commons Attribution License (CC-BY 4.0), which permits any use, distribution and reproduction in any medium, provided the original author(s) and source are credited. 


\section{References}

[1] J.C. Pati, A. Salam and J.A. Strathdee, Are quarks composite?, Phys. Lett. 59B (1975) 265 [INSPIRE].

[2] H. Terazawa, M. Yasuè, K. Akama and M. Hayashi, Observable effects of the possible substructure of leptons and quarks, Phys. Lett. 112B (1982) 387 [INSPIRE].

[3] E. Eichten, K.D. Lane and M.E. Peskin, New tests for quark and lepton substructure, Phys. Rev. Lett. 50 (1983) 811 [INSPIRE].

[4] H. Harari, Composite models for quarks and leptons, Phys. Rept. 104 (1984) 159 [INSPIRE].

[5] K.D. Lane, F.E. Paige, T. Skwarnicki and W.J. Womersley, Simulations of supercollider physics, Phys. Rept. 278 (1997) 291 [hep-ph/9412280] [INSPIRE].

[6] U. Baur, M. Spira and P.M. Zerwas, Excited quark and lepton production at hadron colliders, Phys. Rev. D 42 (1990) 815 [INSPIRE].

[7] O.W. Greenberg and C.A. Nelson, Composite models of leptons, Phys. Rev. D 10 (1974) 2567 [INSPIRE].

[8] O.W. Greenberg and J. Sucher, A quantum structure dynamic model of quarks, leptons, weak vector bosons and Higgs mesons, Phys. Lett. 99B (1981) 339 [INSPIRE].

[9] S. Biondini, R. Leonardi, O. Panella and M. Presilla, Perturbative unitarity bounds for effective composite models, Phys. Lett. B 795 (2019) 644.

[10] ALEPH collaboration, Search for excited leptons at 130 GeV-140 GeV, Phys. Lett. B 385 (1996) 445 [INSPIRE].

[11] DELPHI collaboration, Search for composite and exotic fermions at LEP-2, Eur. Phys. J. C 8 (1999) 41 [hep-ex/9811005] [INSPIRE].

[12] OPAL collaboration, Search for unstable heavy and excited leptons at LEP 2, Eur. Phys. J. C 14 (2000) 73 [hep-ex/0001056] [InSPIRE].

[13] L3 collaboration, Search for excited leptons at LEP, Phys. Lett. B 568 (2003) 23 [hep-ex/0306016] [INSPIRE].

[14] H1 collaboration, Search for excited electrons in ep collisions at HERA, Phys. Lett. B 666 (2008) 131 [arXiv:0805.4530] [INSPIRE].

[15] CDF collaboration, Search for excited and exotic electrons in the er decay channel in $p \bar{p}$ collisions at $\sqrt{s}=1.96$ TeV, Phys. Rev. Lett. 94 (2005) 101802 [hep-ex/0410013] [INSPIRE].

[16] CDF collaboration, Search for excited and exotic muons in the $\mu \gamma$ decay channel in $p \bar{p}$ collisions at $\sqrt{s}=1.96$ TeV, Phys. Rev. Lett. 97 (2006) 191802 [hep-ex/0606043] [INSPIRE].

[17] D0 collaboration, Search for excited muons in $p \bar{p}$ collisions at $\sqrt{s}=1.96$ TeV, Phys. Rev. D 73 (2006) 111102 [hep-ex/0604040] [INSPIRE].

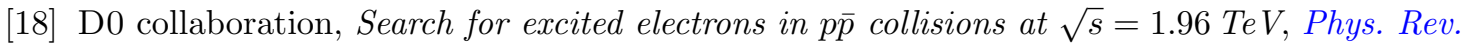
D 77 (2008) 091102 [arXiv:0801.0877] [INSPIRE].

[19] ATLAS collaboration, Search for excited electrons and muons in $\sqrt{s}=8$ TeV proton-proton collisions with the ATLAS detector, New J. Phys. 15 (2013) 093011 [arXiv:1308.1364] [INSPIRE].

[20] ATLAS collaboration, A search for an excited muon decaying to a muon and two jets in $p p$ collisions at $\sqrt{s}=8 \mathrm{TeV}$ with the ATLAS detector, New J. Phys. 18 (2016) 073021 [Erratum ibid. 21 (2019) 109501] [arXiv:1601.05627] [INSPIRE]. 
[21] ATLAS collaboration, Search for excited electrons singly produced in proton-proton collisions at $\sqrt{s}=13 \mathrm{TeV}$ with the ATLAS experiment at the LHC, Eur. Phys. J. C 79 (2019) 803 [arXiv: 1906. 03204] [INSPIRE].

[22] CMS collaboration, Search for excited leptons in pp collisions at $\sqrt{s}=7$ TeV, Phys. Lett. B 720 (2013) 309 [arXiv: 1210.2422] [INSPIRE].

[23] CMS collaboration, Search for excited leptons in proton-proton collisions at $\sqrt{s}=8 \mathrm{TeV}$, JHEP 03 (2016) 125 [arXiv: 1511.01407] [INSPIRE].

[24] CMS collaboration, Search for excited leptons in $\ell \ell \gamma$ final states in proton-proton collisions at $\sqrt{s}=13 \mathrm{TeV}$, JHEP 04 (2019) 015 [arXiv: 1811.03052] [INSPIRE].

[25] CMS collaboration, Performance of photon reconstruction and identification with the CMS detector in proton-proton collisions at $\sqrt{s}=8 \mathrm{TeV}, 2015$ JINST $10 \mathrm{P} 08010$ [arXiv: 1502.02702] [INSPIRE].

[26] CMS collaboration, Search for heavy narrow dilepton resonances in pp collisions at $\sqrt{s}=7$ $\mathrm{TeV}$ and $\sqrt{s}=8 \mathrm{TeV}$, Phys. Lett. B 720 (2013) 63 [arXiv:1212.6175] [INSPIRE].

[27] CMS collaboration, Performance of the CMS muon detector and muon reconstruction with proton-proton collisions at $\sqrt{s}=13 \mathrm{TeV}, 2018$ JINST 13 P06015 [arXiv: 1804.04528] [INSPIRE].

[28] CMS collaboration, Particle-flow reconstruction and global event description with the CMS detector, 2017 JINST 12 P10003 [arXiv:1706.04965] [INSPIRE].

[29] CMS collaboration, The CMS trigger system, 2017 JINST 12 P01020 [arXiv:1609.02366] [INSPIRE].

[30] CMS collaboration, The CMS experiment at the CERN LHC, 2008 JINST 3 S08004 [INSPIRE].

[31] T. Sjöstrand et al., An introduction to PYTHIA 8.2, Comput. Phys. Commun. 191 (2015) 159 [arXiv: 1410.3012] [INSPIRE].

[32] R.D. Ball et al., Parton distributions with LHC data, Nucl. Phys. B 867 (2013) 244 [arXiv: 1207.1303] [INSPIRE].

[33] NNPDF collaboration, Parton distributions from high-precision collider data, Eur. Phys. J. C 77 (2017) 663 [arXiv:1706.00428] [INSPIRE].

[34] CMS collaboration, Extraction and validation of a new set of CMS PYTHIA8 tunes from underlying-event measurements, Eur. Phys. J. C 80 (2020) 4 [arXiv:1903.12179] [INSPIRE].

[35] M. Cacciari, G.P. Salam and G. Soyez, The anti- $k_{t}$ jet clustering algorithm, JHEP 04 (2008) 063 [arXiv: 0802.1189] [INSPIRE].

[36] M. Cacciari, G.P. Salam and G. Soyez, FastJet user manual, Eur. Phys. J. C 72 (2012) 1896 [arXiv: 1111.6097] [INSPIRE].

[37] CMS collaboration, Search for high-mass resonances in dilepton final states in proton-proton collisions at $\sqrt{s}=13 \mathrm{TeV}$, JHEP 06 (2018) 120 [arXiv:1803.06292] [INSPIRE].

[38] CMS collaboration, Performance of CMS muon reconstruction in pp collision events at $\sqrt{s}=7 \mathrm{TeV}, 2012$ JINST $7 \mathrm{P} 10002$ [arXiv:1206.4071] [INSPIRE].

[39] CMS collaboration, Jet energy scale and resolution in the CMS experiment in pp collisions at $8 \mathrm{TeV}, 2017$ JINST $12 \mathrm{P} 02014$ [arXiv: 1607.03663] [INSPIRE].

[40] J. Alwall et al., The automated computation of tree-level and next-to-leading order differential cross sections and their matching to parton shower simulations, JHEP 07 (2014) 079 [arXiv: 1405.0301] [INSPIRE]. 
[41] R. Frederix and S. Frixione, Merging meets matching in MC@NLO, JHEP 12 (2012) 061 [arXiv: 1209.6215] [INSPIRE].

[42] R. Gavin, Y. Li, F. Petriello and S. Quackenbush, FEWZ 2.0: a code for hadronic Z production at next-to-next-to-leading order, Comput. Phys. Commun. 182 (2011) 2388 [arXiv: 1011.3540] [INSPIRE].

[43] P. Nason, A new method for combining NLO QCD with shower Monte Carlo algorithms, JHEP 11 (2004) 040 [hep-ph/0409146] [INSPIRE].

[44] S. Frixione, P. Nason and C. Oleari, Matching NLO QCD computations with Parton Shower simulations: the POWHEG method, JHEP 11 (2007) 070 [arXiv:0709.2092] [INSPIRE].

[45] S. Alioli, P. Nason, C. Oleari and E. Re, A general framework for implementing NLO calculations in shower Monte Carlo programs: the POWHEG BOX, JHEP 06 (2010) 043 [arXiv: 1002.2581] [INSPIRE].

[46] E. Re, Single-top Wt-channel production matched with parton showers using the POWHEG method, Eur. Phys. J. C 71 (2011) 1547 [arXiv:1009.2450] [INSPIRE].

[47] S. Frixione, P. Nason and G. Ridolfi, A positive-weight next-to-leading-order Monte Carlo for heavy flavour hadroproduction, JHEP 09 (2007) 126 [arXiv:0707.3088] [INSPIRE].

[48] M. Czakon, P. Fiedler and A. Mitov, Total top-quark pair-production cross section at hadron colliders through $O\left(\alpha_{S}^{4}\right)$, Phys. Rev. Lett. 110 (2013) 252004 [arXiv:1303.6254] [INSPIRE].

[49] NNPDF collaboration, Parton distributions for the LHC Run II, JHEP 04 (2015) 040 [arXiv: 1410.8849] [INSPIRE].

[50] GEANT4 collaboration, GEANT4 - a simulation toolkit, Nucl. Instrum. Meth. A 506 (2003) 250 [INSPIRE].

[51] CMS collaboration, Search for high-mass resonances in final states with a lepton and missing transverse momentum at $\sqrt{s}=13 \mathrm{TeV}$, JHEP 06 (2018) 128 [arXiv:1803.11133] [INSPIRE].

[52] CMS collaboration, Performance of electron reconstruction and selection with the CMS detector in proton-proton collisions at $\sqrt{s}=8 \mathrm{TeV}, 2015$ JINST $10 \mathrm{P} 06005$ [arXiv: 1502.02701] [INSPIRE].

[53] CMS collaboration, CMS luminosity measurements for the 2016 data taking period, CMS-PAS-LUM-17-001 (2017).

[54] CMS collaboration, CMS luminosity measurement for the 2017 data-taking period at $\sqrt{s}=13 \mathrm{TeV}$, CMS-PAS-LUM-17-004 (2017).

[55] CMS collaboration, Measurement of the inelastic proton-proton cross section at $\sqrt{s}=13$ TeV, JHEP 07 (2018) 161 [arXiv: 1802.02613] [INSPIRE].

[56] J. Butterworth et al., PDF4LHC recommendations for LHC Run II, J. Phys. G 43 (2016) 023001 [arXiv: 1510.03865] [INSPIRE].

[57] A. Buckley et al., LHAPDF6: parton density access in the LHC precision era, Eur. Phys. J. C 75 (2015) 132 [arXiv: 1412.7420] [InSPIRE].

[58] Particle Data Group collaboration, Review of particle physics, Phys. Rev. D 98 (2018) 030001 [INSPIRE]. 


\section{The CMS collaboration}

Yerevan Physics Institute, Yerevan, Armenia

A.M. Sirunyan ${ }^{\dagger}$, A. Tumasyan

\section{Institut für Hochenergiephysik, Wien, Austria}

W. Adam, F. Ambrogi, T. Bergauer, J. Brandstetter, M. Dragicevic, J. Erö, A. Escalante Del Valle, M. Flechl, R. Frühwirth ${ }^{1}$, M. Jeitler ${ }^{1}$, N. Krammer, I. Krätschmer, D. Liko, T. Madlener, I. Mikulec, N. Rad, J. Schieck ${ }^{1}$, R. Schöfbeck, M. Spanring, D. Spitzbart, W. Waltenberger, C.-E. Wulz ${ }^{1}$, M. Zarucki

Institute for Nuclear Problems, Minsk, Belarus

V. Drugakov, V. Mossolov, J. Suarez Gonzalez

\section{Universiteit Antwerpen, Antwerpen, Belgium}

M.R. Darwish, E.A. De Wolf, D. Di Croce, X. Janssen, J. Lauwers, A. Lelek, M. Pieters, H. Rejeb Sfar, H. Van Haevermaet, P. Van Mechelen, S. Van Putte, N. Van Remortel

\section{Vrije Universiteit Brussel, Brussel, Belgium}

F. Blekman, E.S. Bols, S.S. Chhibra, J. D'Hondt, J. De Clercq, D. Lontkovskyi, S. Lowette, I. Marchesini, S. Moortgat, L. Moreels, Q. Python, K. Skovpen, S. Tavernier, W. Van Doninck, P. Van Mulders, I. Van Parijs

\section{Université Libre de Bruxelles, Bruxelles, Belgium}

D. Beghin, B. Bilin, H. Brun, B. Clerbaux, G. De Lentdecker, H. Delannoy, B. Dorney,

L. Favart, A. Grebenyuk, A.K. Kalsi, J. Luetic, A. Popov, N. Postiau, E. Starling,

L. Thomas, C. Vander Velde, P. Vanlaer, D. Vannerom, Q. Wang

\section{Ghent University, Ghent, Belgium}

T. Cornelis, D. Dobur, I. Khvastunov², C. Roskas, D. Trocino, M. Tytgat, W. Verbeke,

B. Vermassen, M. Vit, N. Zaganidis

Université Catholique de Louvain, Louvain-la-Neuve, Belgium

O. Bondu, G. Bruno, C. Caputo, P. David, C. Delaere, M. Delcourt, A. Giammanco, V. Lemaitre, A. Magitteri, J. Prisciandaro, A. Saggio, M. Vidal Marono, P. Vischia, J. Zobec

Centro Brasileiro de Pesquisas Fisicas, Rio de Janeiro, Brazil

F.L. Alves, G.A. Alves, G. Correia Silva, C. Hensel, A. Moraes, P. Rebello Teles

Universidade do Estado do Rio de Janeiro, Rio de Janeiro, Brazil

E. Belchior Batista Das Chagas, W. Carvalho, J. Chinellato ${ }^{3}$, E. Coelho, E.M. Da Costa, G.G. Da Silveira ${ }^{4}$, D. De Jesus Damiao, C. De Oliveira Martins, S. Fonseca De Souza, L.M. Huertas Guativa, H. Malbouisson, J. Martins ${ }^{5}$, D. Matos Figueiredo, M. Medina Jaime ${ }^{6}$, M. Melo De Almeida, C. Mora Herrera, L. Mundim, H. Nogima, W.L. Prado Da Silva, L.J. Sanchez Rosas, A. Santoro, A. Sznajder, M. Thiel, E.J. Tonelli Manganote ${ }^{3}$, F. Torres Da Silva De Araujo, A. Vilela Pereira 
Universidade Estadual Paulista ${ }^{a}$, Universidade Federal do $\mathrm{ABC}^{b}$, São Paulo, Brazil

S. Ahuja ${ }^{a}$, C.A. Bernardes ${ }^{a}$, L. Calligaris ${ }^{a}$, T.R. Fernandez Perez Tomei ${ }^{a}$, E.M. Gregores ${ }^{b}$, D.S. Lemos, P.G. Mercadante ${ }^{b}$, S.F. Novaes ${ }^{a}$, SandraS. Padula ${ }^{a}$

Institute for Nuclear Research and Nuclear Energy, Bulgarian Academy of Sciences, Sofia, Bulgaria

A. Aleksandrov, G. Antchev, R. Hadjiiska, P. Iaydjiev, A. Marinov, M. Misheva, M. Rodozov, M. Shopova, G. Sultanov

University of Sofia, Sofia, Bulgaria

M. Bonchev, A. Dimitrov, T. Ivanov, L. Litov, B. Pavlov, P. Petkov

Beihang University, Beijing, China

W. Fang $^{7}$, X. $\mathrm{Gao}^{7}$, L. Yuan

Department of Physics, Tsinghua University, Beijing, China

Z. Hu, Y. Wang

Institute of High Energy Physics, Beijing, China

M. Ahmad, G.M. Chen, H.S. Chen, M. Chen, C.H. Jiang, D. Leggat, H. Liao, Z. Liu, S.M. Shaheen ${ }^{8}$, A. Spiezia, J. Tao, E. Yazgan, H. Zhang, S. Zhang ${ }^{8}$, J. Zhao

State Key Laboratory of Nuclear Physics and Technology, Peking University, Beijing, China

A. Agapitos, Y. Ban, G. Chen, A. Levin, J. Li, L. Li, Q. Li, Y. Mao, S.J. Qian, D. Wang

Universidad de Los Andes, Bogota, Colombia

C. Avila, A. Cabrera, L.F. Chaparro Sierra, C. Florez, C.F. González Hernández, M.A. Segura Delgado

Universidad de Antioquia, Medellin, Colombia

J. Mejia Guisao, J.D. Ruiz Alvarez, C.A. Salazar González, N. Vanegas Arbelaez

University of Split, Faculty of Electrical Engineering, Mechanical Engineering and Naval Architecture, Split, Croatia

D. Giljanović, N. Godinovic, D. Lelas, I. Puljak, T. Sculac

University of Split, Faculty of Science, Split, Croatia

Z. Antunovic, M. Kovac

Institute Rudjer Boskovic, Zagreb, Croatia

V. Brigljevic, S. Ceci, D. Ferencek, K. Kadija, B. Mesic, M. Roguljic, A. Starodumov ${ }^{9}$,

T. Susa

University of Cyprus, Nicosia, Cyprus

M.W. Ather, A. Attikis, E. Erodotou, A. Ioannou, M. Kolosova, S. Konstantinou,

G. Mavromanolakis, J. Mousa, C. Nicolaou, F. Ptochos, P.A. Razis, H. Rykaczewski,

D. Tsiakkouri 
Charles University, Prague, Czech Republic

M. Finger ${ }^{10}$, M. Finger Jr. ${ }^{10}$, A. Kveton, J. Tomsa

Escuela Politecnica Nacional, Quito, Ecuador

E. Ayala

Universidad San Francisco de Quito, Quito, Ecuador

E. Carrera Jarrin

Academy of Scientific Research and Technology of the Arab Republic of Egypt, Egyptian Network of High Energy Physics, Cairo, Egypt

Y. Assran ${ }^{11,12}$, S. Elgammal ${ }^{12}$

National Institute of Chemical Physics and Biophysics, Tallinn, Estonia

S. Bhowmik, A. Carvalho Antunes De Oliveira, R.K. Dewanjee, K. Ehataht, M. Kadastik, M. Raidal, C. Veelken

Department of Physics, University of Helsinki, Helsinki, Finland

P. Eerola, L. Forthomme, H. Kirschenmann, K. Osterberg, M. Voutilainen

Helsinki Institute of Physics, Helsinki, Finland

F. Garcia, J. Havukainen, J.K. Heikkilä, T. Järvinen, V. Karimäki, R. Kinnunen,

T. Lampén, K. Lassila-Perini, S. Laurila, S. Lehti, T. Lindén, P. Luukka, T. Mäenpää,

H. Siikonen, E. Tuominen, J. Tuominiemi

Lappeenranta University of Technology, Lappeenranta, Finland

T. Tuuva

IRFU, CEA, Université Paris-Saclay, Gif-sur-Yvette, France

M. Besancon, F. Couderc, M. Dejardin, D. Denegri, B. Fabbro, J.L. Faure, F. Ferri, S. Ganjour, A. Givernaud, P. Gras, G. Hamel de Monchenault, P. Jarry, C. Leloup, E. Locci, J. Malcles, J. Rander, A. Rosowsky, M.Ö. Sahin, A. Savoy-Navarro ${ }^{13}$, M. Titov

Laboratoire Leprince-Ringuet, CNRS/IN2P3, Ecole Polytechnique, Institut Polytechnique de Paris

C. Amendola, F. Beaudette, P. Busson, C. Charlot, B. Diab, G. Falmagne, R. Granier de Cassagnac, I. Kucher, A. Lobanov, C. Martin Perez, M. Nguyen, C. Ochando, P. Paganini, J. Rembser, R. Salerno, J.B. Sauvan, Y. Sirois, A. Zabi, A. Zghiche

Université de Strasbourg, CNRS, IPHC UMR 7178, Strasbourg, France

J.-L. Agram ${ }^{14}$, J. Andrea, D. Bloch, G. Bourgatte, J.-M. Brom, E.C. Chabert, C. Collard, E. Conte ${ }^{14}$, J.-C. Fontaine ${ }^{14}$, D. Gelé, U. Goerlach, M. Jansová, A.-C. Le Bihan, N. Tonon, P. Van Hove

Centre de Calcul de l'Institut National de Physique Nucleaire et de Physique des Particules, CNRS/IN2P3, Villeurbanne, France

S. Gadrat 
Université de Lyon, Université Claude Bernard Lyon 1, CNRS-IN2P3, Institut de Physique Nucléaire de Lyon, Villeurbanne, France

S. Beauceron, C. Bernet, G. Boudoul, C. Camen, N. Chanon, R. Chierici, D. Contardo, P. Depasse, H. El Mamouni, J. Fay, S. Gascon, M. Gouzevitch, B. Ille, Sa. Jain, F. Lagarde, I.B. Laktineh, H. Lattaud, M. Lethuillier, L. Mirabito, S. Perries, V. Sordini, G. Touquet, M. Vander Donckt, S. Viret

Georgian Technical University, Tbilisi, Georgia

T. Toriashvili ${ }^{15}$

Tbilisi State University, Tbilisi, Georgia

Z. Tsamalaidze ${ }^{10}$

RWTH Aachen University, I. Physikalisches Institut, Aachen, Germany

C. Autermann, L. Feld, M.K. Kiesel, K. Klein, M. Lipinski, D. Meuser, A. Pauls, M. Preuten, M.P. Rauch, C. Schomakers, J. Schulz, M. Teroerde, B. Wittmer

RWTH Aachen University, III. Physikalisches Institut A, Aachen, Germany

A. Albert, M. Erdmann, S. Erdweg, T. Esch, B. Fischer, R. Fischer, S. Ghosh, T. Hebbeker, K. Hoepfner, H. Keller, L. Mastrolorenzo, M. Merschmeyer, A. Meyer, P. Millet, G. Mocellin, S. Mondal, S. Mukherjee, D. Noll, A. Novak, T. Pook, A. Pozdnyakov, T. Quast, M. Radziej, Y. Rath, H. Reithler, M. Rieger, J. Roemer, A. Schmidt, S.C. Schuler, A. Sharma, S. Thüer, S. Wiedenbeck

RWTH Aachen University, III. Physikalisches Institut B, Aachen, Germany G. Flügge, W. Haj Ahmad ${ }^{16}$, O. Hlushchenko, T. Kress, T. Müller, A. Nehrkorn, A. Nowack, C. Pistone, O. Pooth, D. Roy, H. Sert, A. Stahl ${ }^{17}$

Deutsches Elektronen-Synchrotron, Hamburg, Germany

M. Aldaya Martin, P. Asmuss, I. Babounikau, H. Bakhshiansohi, K. Beernaert, O. Behnke, U. Behrens, A. Bermúdez Martínez, D. Bertsche, A.A. Bin Anuar, K. Borras ${ }^{18}$, V. Botta, A. Campbell, A. Cardini, P. Connor, S. Consuegra Rodríguez, C. Contreras-Campana, V. Danilov, A. De Wit, M.M. Defranchis, C. Diez Pardos, D. Domínguez Damiani, G. Eckerlin, D. Eckstein, T. Eichhorn, A. Elwood, E. Eren, E. Gallo ${ }^{19}$, A. Geiser, J.M. Grados Luyando, A. Grohsjean, M. Guthoff, M. Haranko, A. Harb, A. Jafari, N.Z. Jomhari, H. Jung, A. Kasem ${ }^{18}$, M. Kasemann, H. Kaveh, J. Keaveney, C. Kleinwort, J. Knolle, D. Krücker, W. Lange, T. Lenz, J. Leonard, J. Lidrych, K. Lipka, W. Lohmann ${ }^{20}$, R. Mankel, I.-A. Melzer-Pellmann, A.B. Meyer, M. Meyer, M. Missiroli, G. Mittag, J. Mnich, A. Mussgiller, V. Myronenko, D. Pérez Adán, S.K. Pflitsch, D. Pitzl, A. Raspereza, A. Saibel, M. Savitskyi, V. Scheurer, P. Schütze, C. Schwanenberger, R. Shevchenko, A. Singh, H. Tholen, O. Turkot, A. Vagnerini, M. Van De Klundert, G.P. Van Onsem, R. Walsh, Y. Wen, K. Wichmann, C. Wissing, O. Zenaiev, R. Zlebcik

University of Hamburg, Hamburg, Germany

R. Aggleton, S. Bein, L. Benato, A. Benecke, V. Blobel, T. Dreyer, A. Ebrahimi, A. Fröhlich, C. Garbers, E. Garutti, D. Gonzalez, P. Gunnellini, J. Haller, A. Hinzmann, A. Karavdina, G. Kasieczka, R. Klanner, R. Kogler, N. Kovalchuk, S. Kurz, V. Kutzner, 
J. Lange, T. Lange, A. Malara, D. Marconi, J. Multhaup, M. Niedziela, C.E.N. Niemeyer, D. Nowatschin, A. Perieanu, A. Reimers, O. Rieger, C. Scharf, P. Schleper, S. Schumann, J. Schwandt, J. Sonneveld, H. Stadie, G. Steinbrück, F.M. Stober, M. Stöver, B. Vormwald, I. Zoi

Karlsruher Institut fuer Technologie, Karlsruhe, Germany

M. Akbiyik, C. Barth, M. Baselga, S. Baur, T. Berger, E. Butz, R. Caspart, T. Chwalek, W. De Boer, A. Dierlamm, K. El Morabit, N. Faltermann, M. Giffels, P. Goldenzweig, A. Gottmann, M.A. Harrendorf, F. Hartmann ${ }^{17}$, U. Husemann, S. Kudella, S. Mitra, M.U. Mozer, Th. Müller, M. Musich, A. Nürnberg, G. Quast, K. Rabbertz, M. Schröder, I. Shvetsov, H.J. Simonis, R. Ulrich, M. Weber, C. Wöhrmann, R. Wolf

Institute of Nuclear and Particle Physics (INPP), NCSR Demokritos, Aghia Paraskevi, Greece

G. Anagnostou, P. Asenov, G. Daskalakis, T. Geralis, A. Kyriakis, D. Loukas, G. Paspalaki National and Kapodistrian University of Athens, Athens, Greece

M. Diamantopoulou, G. Karathanasis, P. Kontaxakis, A. Panagiotou, I. Papavergou, N. Saoulidou, A. Stakia, K. Theofilatos, K. Vellidis

National Technical University of Athens, Athens, Greece

G. Bakas, K. Kousouris, I. Papakrivopoulos, G. Tsipolitis

University of Ioánnina, Ioánnina, Greece

I. Evangelou, C. Foudas, P. Gianneios, P. Katsoulis, P. Kokkas, S. Mallios, K. Manitara, N. Manthos, I. Papadopoulos, J. Strologas, F.A. Triantis, D. Tsitsonis

MTA-ELTE Lendület CMS Particle and Nuclear Physics Group, Eötvös Loránd University, Budapest, Hungary

M. Bartók ${ }^{21}$, M. Csanad, P. Major, K. Mandal, A. Mehta, M.I. Nagy, G. Pasztor, O. Surányi, G.I. Veres

Wigner Research Centre for Physics, Budapest, Hungary

G. Bencze, C. Hajdu, D. Horvath ${ }^{22}$, F. Sikler, T.Á. Vámi, V. Veszpremi, G. Vesztergombi ${ }^{\dagger}$

Institute of Nuclear Research ATOMKI, Debrecen, Hungary

N. Beni, S. Czellar, J. Karancsi ${ }^{21}$, A. Makovec, J. Molnar, Z. Szillasi

Institute of Physics, University of Debrecen, Debrecen, Hungary

P. Raics, D. Teyssier, Z.L. Trocsanyi, B. Ujvari

Eszterhazy Karoly University, Karoly Robert Campus, Gyongyos, Hungary

T. Csorgo, W.J. Metzger, F. Nemes, T. Novak

Indian Institute of Science (IISc), Bangalore, India

S. Choudhury, J.R. Komaragiri, P.C. Tiwari 
National Institute of Science Education and Research, HBNI, Bhubaneswar, India

S. Bahinipati ${ }^{24}$, C. Kar, G. Kole, P. Mal, V.K. Muraleedharan Nair Bindhu, A. Nayak ${ }^{25}$, D.K. Sahoo ${ }^{24}$, S.K. Swain

Panjab University, Chandigarh, India

S. Bansal, S.B. Beri, V. Bhatnagar, S. Chauhan, R. Chawla, N. Dhingra, R. Gupta,

A. Kaur, M. Kaur, S. Kaur, P. Kumari, M. Lohan, M. Meena, K. Sandeep, S. Sharma, J.B. Singh, A.K. Virdi, G. Walia

University of Delhi, Delhi, India

A. Bhardwaj, B.C. Choudhary, R.B. Garg, M. Gola, S. Keshri, Ashok Kumar, S. Malhotra, M. Naimuddin, P. Priyanka, K. Ranjan, Aashaq Shah, R. Sharma

Saha Institute of Nuclear Physics, HBNI, Kolkata, India

R. Bhardwaj ${ }^{26}$, M. Bharti ${ }^{26}$, R. Bhattacharya, S. Bhattacharya, U. Bhawandeep ${ }^{26}$, D. Bhowmik, S. Dey, S. Dutta, S. Ghosh, M. Maity ${ }^{27}$, K. Mondal, S. Nandan, A. Purohit, P.K. Rout, G. Saha, S. Sarkar, T. Sarkar ${ }^{27}$, M. Sharan, B. Singh ${ }^{26}$, S. Thakur ${ }^{26}$

Indian Institute of Technology Madras, Madras, India

P.K. Behera, P. Kalbhor, A. Muhammad, P.R. Pujahari, A. Sharma, A.K. Sikdar

Bhabha Atomic Research Centre, Mumbai, India

R. Chudasama, D. Dutta, V. Jha, V. Kumar, D.K. Mishra, P.K. Netrakanti, L.M. Pant, P. Shukla

Tata Institute of Fundamental Research-A, Mumbai, India

T. Aziz, M.A. Bhat, S. Dugad, G.B. Mohanty, N. Sur, RavindraKumar Verma

Tata Institute of Fundamental Research-B, Mumbai, India

S. Banerjee, S. Bhattacharya, S. Chatterjee, P. Das, M. Guchait, S. Karmakar, S. Kumar, G. Majumder, K. Mazumdar, N. Sahoo, S. Sawant

Indian Institute of Science Education and Research (IISER), Pune, India

S. Chauhan, S. Dube, V. Hegde, A. Kapoor, K. Kothekar, S. Pandey, A. Rane, A. Rastogi,

S. Sharma

Institute for Research in Fundamental Sciences (IPM), Tehran, Iran

S. Chenarani ${ }^{28}$, E. Eskandari Tadavani, S.M. Etesami ${ }^{28}$, M. Khakzad, M. Mohammadi Najafabadi, M. Naseri, F. Rezaei Hosseinabadi

University College Dublin, Dublin, Ireland

M. Felcini, M. Grunewald

INFN Sezione di Bari $^{a}$, Università di Bari ${ }^{b}$, Politecnico di Bari ${ }^{c}$, Bari, Italy M. Abbrescia ${ }^{a, b}$, C. Calabria ${ }^{a, b}$, A. Colaleo $^{a}$, D. Creanza $^{a, c}$, L. Cristella $^{a, b}$, N. De Filippis $^{a, c}$, M. De Palma ${ }^{a, b}$, A. Di Florio ${ }^{a, b}$, L. Fiore $^{a}$, A. Gelmi ${ }^{a, b}$, G. Iaselli ${ }^{a, c}$, M. Ince ${ }^{a, b}$, S. Lezki $^{a, b}$, G. Maggi ${ }^{a, c}$, M. Maggi ${ }^{a}$, G. Miniello ${ }^{a, b}$, S. My ${ }^{a, b}$, S. Nuzzo $^{a, b}$, A. Pompili ${ }^{a, b}$, G. Pugliese ${ }^{a, c}$,

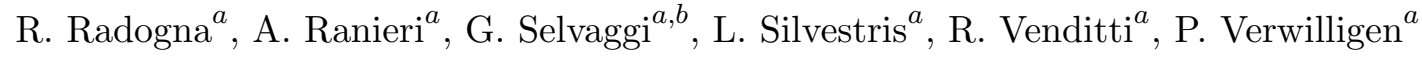


INFN Sezione di Bologna ${ }^{a}$, Università di Bologna ${ }^{b}$, Bologna, Italy

G. Abbiendi ${ }^{a}$, C. Battilana ${ }^{a, b}$, D. Bonacorsi ${ }^{a, b}$, L. Borgonovi $^{a, b}$, S. Braibant-Giacomelli $^{a, b}$, R. Campanini ${ }^{a, b}$, P. Capiluppi ${ }^{a, b}$, A. Castro ${ }^{a, b}$, F.R. Cavallo ${ }^{a}$, C. Ciocca ${ }^{a}$, G. Codispoti $^{a, b}$, M. Cuffiani ${ }^{a, b}$, G.M. Dallavalle ${ }^{a}$, F. Fabbri ${ }^{a}$, A. Fanfani ${ }^{a, b}$, E. Fontanesi, P. Giacomelli ${ }^{a}$, C. Grandi ${ }^{a}$, L. Guiducci ${ }^{a, b}$, F. Iemmi ${ }^{a, b}$, S. Lo Meo ${ }^{a, 29}$, S. Marcellini ${ }^{a}$, G. Masetti ${ }^{a}$,

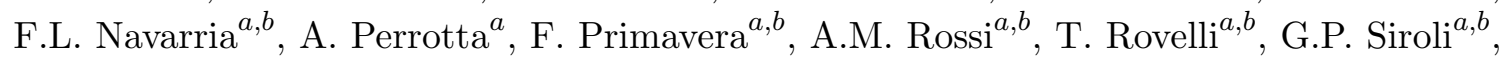
N. $\operatorname{Tosi}^{a}$

INFN Sezione di Catania ${ }^{a}$, Università di Catania ${ }^{b}$, Catania, Italy

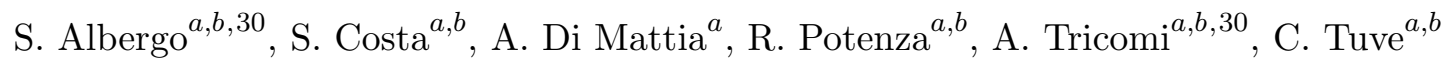

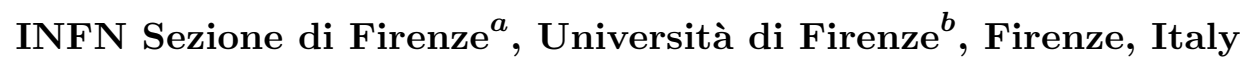

G. Barbagli ${ }^{a}$, R. Ceccarelli, K. Chatterjee ${ }^{a, b}$, V. Ciulli ${ }^{a, b}$, C. Civinini ${ }^{a}$, R. D'Alessandro ${ }^{a, b}{ }^{,}$ E. Focardi ${ }^{a, b}$, G. Latino, P. Lenzi ${ }^{a, b}$, M. Meschini $^{a}$, S. Paoletti $^{a}$, G. Sguazzoni ${ }^{a}$, D. Strom ${ }^{a}$, L. Viliani ${ }^{a}$

INFN Laboratori Nazionali di Frascati, Frascati, Italy

L. Benussi, S. Bianco, D. Piccolo

INFN Sezione di Genova ${ }^{a}$, Università di Genova ${ }^{b}$, Genova, Italy

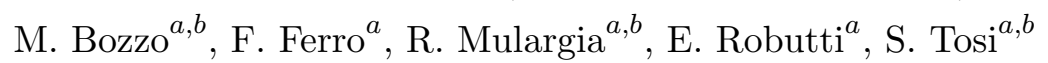

INFN Sezione di Milano-Bicocca $^{a}$, Università di Milano-Bicocca ${ }^{b}$, Milano, Italy

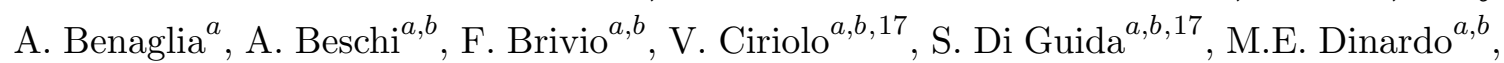

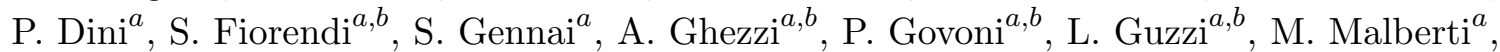
S. Malvezzi ${ }^{a}$, D. Menasce ${ }^{a}$, F. Monti ${ }^{a, b}$, L. Moroni ${ }^{a}$, G. Ortona ${ }^{a, b}$, M. Paganoni $^{a, b}$, D. Pedrini $^{a}$, S. Ragazzi ${ }^{a, b}$, T. Tabarelli de Fatis ${ }^{a, b}$, D. Zuolo ${ }^{a, b}$

INFN Sezione di Napoli ${ }^{a}$, Università di Napoli 'Federico II ${ }^{b}$, Napoli, Italy, Università della Basilicata $^{c}$, Potenza, Italy, Università G. Marconi ${ }^{d}$, Roma, Italy

S. Buontempo ${ }^{a}$, N. Cavallo $^{a, c}$, A. De Iorio ${ }^{a, b}$, A. Di Crescenzo ${ }^{a, b}$, F. Fabozzi $^{a, c}$, F. Fienga $^{a}$,

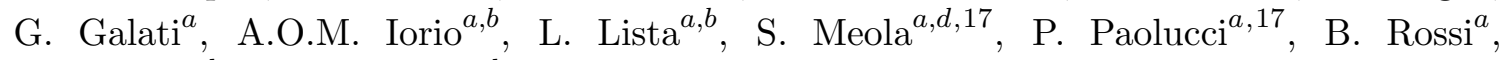
C. Sciacca ${ }^{a, b}$, E. Voevodina ${ }^{a, b}$

INFN Sezione di Padova ${ }^{a}$, Università di Padova ${ }^{b}$, Padova, Italy, Università di Trento $^{c}$, Trento, Italy

P. Azzi ${ }^{a}$, N. Bacchetta ${ }^{a}$, D. Bisello ${ }^{a, b}$, A. Boletti ${ }^{a, b}$, A. Bragagnolo, R. Carlin ${ }^{a, b}$, P. Checchia ${ }^{a}$, P. De Castro Manzano ${ }^{a}$, T. Dorigo ${ }^{a}$, U. Dosselli ${ }^{a}$, F. Gasparini ${ }^{a, b}$, U. Gasparini $^{a, b}$, A. Gozzelino ${ }^{a}$, S.Y. Hoh, P. Lujan, M. Margoni ${ }^{a, b}$, A.T. Meneguzzo ${ }^{a, b}$,

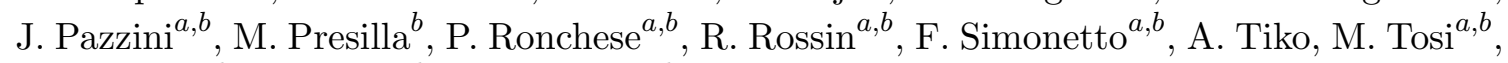
M. Zanetti ${ }^{a, b}$, P. Zotto ${ }^{a, b}$, G. Zumerle ${ }^{a, b}$

INFN Sezione di Pavia ${ }^{a}$, Università di Pavia ${ }^{b}$, Pavia, Italy

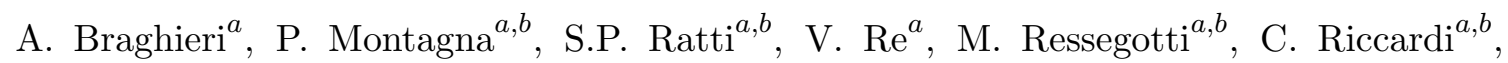
P. Salvini ${ }^{a}$, I. Vai ${ }^{a, b}$, P. Vitulo ${ }^{a, b}$ 
INFN Sezione di Perugia ${ }^{a}$, Università di Perugia ${ }^{b}$, Perugia, Italy

M. Biasini ${ }^{a, b}$, G.M. Bilei ${ }^{a}$, C. Cecchi ${ }^{a, b}$, D. Ciangottini ${ }^{a, b}$, L. Fanò ${ }^{a, b}$, P. Lariccia ${ }^{a, b}$,

R. Leonardi ${ }^{a, b}$, E. Manoni ${ }^{a}$, G. Mantovani ${ }^{a, b}$, V. Mariani ${ }^{a, b}$, M. Menichelli $^{a}$, A. Rossi $^{a, b}$,

A. Santocchia ${ }^{a, b}$, D. Spiga ${ }^{a}$

INFN Sezione di Pisa ${ }^{a}$, Università di Pisa ${ }^{b}$, Scuola Normale Superiore di Pisa ${ }^{c}$, Pisa, Italy

K. $\operatorname{Androsov}^{a}$, P. Azzurri ${ }^{a}$, G. Bagliesi ${ }^{a}$, V. Bertacchi ${ }^{a, c}$, L. Bianchini ${ }^{a}$, T. Boccali ${ }^{a}$,

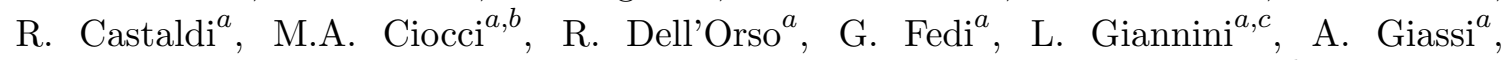
M.T. Grippo ${ }^{a}$, F. Ligabue ${ }^{a, c}$, E. Manca ${ }^{a, c}$, G. Mandorli ${ }^{a, c}$, A. Messineo ${ }^{a, b}$, F. Palla $^{a}$, A. Rizzi ${ }^{a, b}$, G. Rolandi ${ }^{31}$, S. Roy Chowdhury, A. Scribano ${ }^{a}$, P. Spagnolo $^{a}$, R. Tenchini $^{a}$, G. Tonelli ${ }^{a, b}$, N. Turini, A. Venturi ${ }^{a}$, P.G. Verdini ${ }^{a}$

INFN Sezione di Roma ${ }^{a}$, Sapienza Università di Roma ${ }^{b}$, Rome, Italy

F. Cavallari ${ }^{a}$, M. Cipriani ${ }^{a, b}$, D. Del Re ${ }^{a, b}$, E. Di Marco ${ }^{a, b}$, M. Diemoz $^{a}$, E. Longo ${ }^{a, b}$,

B. Marzocchi $^{a, b}$, P. Meridiani ${ }^{a}$, G. Organtini ${ }^{a, b}$, F. Pandolfi ${ }^{a}$, R. Paramatti ${ }^{a, b}$,

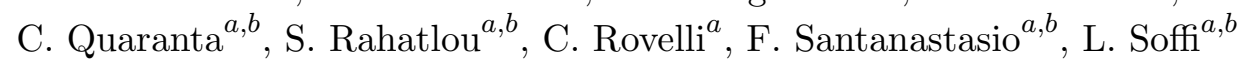

INFN Sezione di Torino ${ }^{a}$, Università di Torino ${ }^{b}$, Torino, Italy, Università del Piemonte Orientale ${ }^{c}$, Novara, Italy

N. Amapane ${ }^{a, b}$, R. Arcidiacono ${ }^{a, c}, \mathrm{~S}$. Argiro ${ }^{a, b}$, M. Arneodo $^{a, c}$, N. Bartosik ${ }^{a}$, R. Bellan $^{a, b}$,

C. Biino ${ }^{a}$, A. Cappati ${ }^{a, b}$, N. Cartiglia $^{a}$, S. Cometti ${ }^{a}$, M. Costa ${ }^{a, b}$, R. Covarelli ${ }^{a, b}$,

N. Demaria ${ }^{a}$, B. Kiani ${ }^{a, b}$, C. Mariotti $^{a}$, S. Maselli ${ }^{a}$, E. Migliore ${ }^{a, b}$, V. Monaco ${ }^{a, b}$,

E. Monteil ${ }^{a, b}$, M. Monteno $^{a}$, M.M. Obertino ${ }^{a, b}$, L. Pacher $^{a, b}$, N. Pastrone $^{a}$, M. Pelliccioni ${ }^{a}$, G.L. Pinna Angioni ${ }^{a, b}$, A. Romero ${ }^{a, b}$, M. Ruspa $^{a, c}$, R. Sacchi ${ }^{a, b}$, R. Salvatico $^{a, b}$, V. Sola $^{a}$, A. Solano ${ }^{a, b}$, D. Soldi ${ }^{a, b}$, A. Staiano ${ }^{a}$

INFN Sezione di Trieste ${ }^{a}$, Università di Trieste ${ }^{b}$, Trieste, Italy

S. Belforte ${ }^{a}$, V. Candelise ${ }^{a, b}$, M. Casarsa ${ }^{a}$, F. Cossutti ${ }^{a}$, A. Da Rold ${ }^{a, b}$, G. Della Ricca ${ }^{a, b}$, F. Vazzoler ${ }^{a, b}$, A. Zanetti ${ }^{a}$

Kyungpook National University, Daegu, Korea

B. Kim, D.H. Kim, G.N. Kim, M.S. Kim, J. Lee, S.W. Lee, C.S. Moon, Y.D. Oh, S.I. Pak,

S. Sekmen, D.C. Son, Y.C. Yang

Chonnam National University, Institute for Universe and Elementary Particles, Kwangju, Korea

H. Kim, D.H. Moon, G. Oh

Hanyang University, Seoul, Korea

B. Francois, T.J. Kim, J. Park

Korea University, Seoul, Korea

S. Cho, S. Choi, Y. Go, D. Gyun, S. Ha, B. Hong, K. Lee, K.S. Lee, J. Lim, J. Park, S.K. Park, Y. Roh

Kyung Hee University, Department of Physics

J. Goh 
Sejong University, Seoul, Korea

H.S. Kim

Seoul National University, Seoul, Korea

J. Almond, J.H. Bhyun, J. Choi, S. Jeon, J. Kim, J.S. Kim, H. Lee, K. Lee, S. Lee, K. Nam, M. Oh, S.B. Oh, B.C. Radburn-Smith, U.K. Yang, H.D. Yoo, I. Yoon, G.B. Yu

University of Seoul, Seoul, Korea

D. Jeon, H. Kim, J.H. Kim, J.S.H. Lee, I.C. Park, I. Watson

Sungkyunkwan University, Suwon, Korea

Y. Choi, C. Hwang, Y. Jeong, J. Lee, Y. Lee, I. Yu

Riga Technical University, Riga, Latvia

V. Veckalns ${ }^{32}$

Vilnius University, Vilnius, Lithuania

V. Dudenas, A. Juodagalvis, G. Tamulaitis, J. Vaitkus

National Centre for Particle Physics, Universiti Malaya, Kuala Lumpur, Malaysia

Z.A. Ibrahim, F. Mohamad Idris ${ }^{33}$, W.A.T. Wan Abdullah, M.N. Yusli, Z. Zolkapli

Universidad de Sonora (UNISON), Hermosillo, Mexico

J.F. Benitez, A. Castaneda Hernandez, J.A. Murillo Quijada, L. Valencia Palomo

Centro de Investigacion y de Estudios Avanzados del IPN, Mexico City, Mexico

H. Castilla-Valdez, E. De La Cruz-Burelo, I. Heredia-De La Cruz ${ }^{34}$, R. Lopez-Fernandez,

A. Sanchez-Hernandez

Universidad Iberoamericana, Mexico City, Mexico

S. Carrillo Moreno, C. Oropeza Barrera, M. Ramirez-Garcia, F. Vazquez Valencia

Benemerita Universidad Autonoma de Puebla, Puebla, Mexico

J. Eysermans, I. Pedraza, H.A. Salazar Ibarguen, C. Uribe Estrada

Universidad Autónoma de San Luis Potosí, San Luis Potosí, Mexico

A. Morelos Pineda

University of Montenegro, Podgorica, Montenegro

N. Raicevic

University of Auckland, Auckland, New Zealand

D. Krofcheck

University of Canterbury, Christchurch, New Zealand

S. Bheesette, P.H. Butler

National Centre for Physics, Quaid-I-Azam University, Islamabad, Pakistan

A. Ahmad, M. Ahmad, Q. Hassan, H.R. Hoorani, W.A. Khan, M.A. Shah, M. Shoaib, M. Waqas 
AGH University of Science and Technology Faculty of Computer Science, Electronics and Telecommunications, Krakow, Poland

V. Avati, L. Grzanka, M. Malawski

National Centre for Nuclear Research, Swierk, Poland

H. Bialkowska, M. Bluj, B. Boimska, M. Górski, M. Kazana, M. Szleper, P. Zalewski

Institute of Experimental Physics, Faculty of Physics, University of Warsaw, Warsaw, Poland

K. Bunkowski, A. Byszuk ${ }^{35}$, K. Doroba, A. Kalinowski, M. Konecki, J. Krolikowski, M. Misiura, M. Olszewski, A. Pyskir, M. Walczak

Laboratório de Instrumentação e Física Experimental de Partículas, Lisboa, Portugal

M. Araujo, P. Bargassa, D. Bastos, A. Di Francesco, P. Faccioli, B. Galinhas, M. Gallinaro, J. Hollar, N. Leonardo, J. Seixas, K. Shchelina, G. Strong, O. Toldaiev, J. Varela

Joint Institute for Nuclear Research, Dubna, Russia

A. Baginyan, Y. Ershov, A. Golunov, I. Golutvin, I. Gorbunov, V. Karjavine, I. Kashunin, V. Korenkov, A. Lanev, A. Malakhov, V. Matveev ${ }^{36,37}$, P. Moisenz, V. Palichik, V. Perelygin, M. Savina, S. Shmatov, S. Shulha, O. Teryaev, N. Voytishin, A. Zarubin

Petersburg Nuclear Physics Institute, Gatchina (St. Petersburg), Russia

L. Chtchipounov, V. Golovtsov, Y. Ivanov, V. Kim ${ }^{38}$, E. Kuznetsova ${ }^{39}$, P. Levchenko, V. Murzin, V. Oreshkin, I. Smirnov, D. Sosnov, V. Sulimov, L. Uvarov, A. Vorobyev

Institute for Nuclear Research, Moscow, Russia

Yu. Andreev, A. Dermenev, S. Gninenko, N. Golubev, A. Karneyeu, M. Kirsanov, N. Krasnikov, A. Pashenkov, D. Tlisov, A. Toropin

Institute for Theoretical and Experimental Physics named by A.I. Alikhanov of NRC 'Kurchatov Institute', Moscow, Russia

V. Epshteyn, V. Gavrilov, N. Lychkovskaya, A. Nikitenko ${ }^{40}$, V. Popov, I. Pozdnyakov, G. Safronov, A. Spiridonov, A. Stepennov, M. Toms, E. Vlasov, A. Zhokin

Moscow Institute of Physics and Technology, Moscow, Russia

T. Aushev

National Research Nuclear University 'Moscow Engineering Physics Institute' (MEPhI), Moscow, Russia

M. Chadeeva ${ }^{41}$, P. Parygin, D. Philippov, E. Popova, V. Rusinov

P.N. Lebedev Physical Institute, Moscow, Russia

V. Andreev, M. Azarkin, I. Dremin, M. Kirakosyan, A. Terkulov

Skobeltsyn Institute of Nuclear Physics, Lomonosov Moscow State University, Moscow, Russia

A. Baskakov, A. Belyaev, E. Boos, V. Bunichev, M. Dubinin ${ }^{42}$, L. Dudko, A. Ershov, V. Klyukhin, O. Kodolova, I. Lokhtin, S. Obraztsov, V. Savrin, A. Snigirev 
Novosibirsk State University (NSU), Novosibirsk, Russia

A. Barnyakov ${ }^{43}$, V. Blinov ${ }^{43}$, T. Dimova ${ }^{43}$, L. Kardapoltsev ${ }^{43}$, Y. Skovpen ${ }^{43}$

Institute for High Energy Physics of National Research Centre 'Kurchatov Institute', Protvino, Russia

I. Azhgirey, I. Bayshev, S. Bitioukov, V. Kachanov, D. Konstantinov, P. Mandrik, V. Petrov, R. Ryutin, S. Slabospitskii, A. Sobol, S. Troshin, N. Tyurin, A. Uzunian, A. Volkov

National Research Tomsk Polytechnic University, Tomsk, Russia

A. Babaev, A. Iuzhakov, V. Okhotnikov

Tomsk State University, Tomsk, Russia

V. Borchsh, V. Ivanchenko, E. Tcherniaev

University of Belgrade: Faculty of Physics and VINCA Institute of Nuclear Sciences

P. Adzic ${ }^{44}$, P. Cirkovic, D. Devetak, M. Dordevic, P. Milenovic, J. Milosevic, M. Stojanovic

Centro de Investigaciones Energéticas Medioambientales y Tecnológicas (CIEMAT), Madrid, Spain

M. Aguilar-Benitez, J. Alcaraz Maestre, A. Álvarez Fernández, I. Bachiller, M. Barrio Luna, J.A. Brochero Cifuentes, C.A. Carrillo Montoya, M. Cepeda, M. Cerrada, N. Colino, B. De La Cruz, A. Delgado Peris, C. Fernandez Bedoya, J.P. Fernández Ramos, J. Flix, M.C. Fouz, O. Gonzalez Lopez, S. Goy Lopez, J.M. Hernandez, M.I. Josa, D. Moran, Á. Navarro Tobar, A. Pérez-Calero Yzquierdo, J. Puerta Pelayo, I. Redondo, L. Romero, S. Sánchez Navas, M.S. Soares, A. Triossi, C. Willmott

Universidad Autónoma de Madrid, Madrid, Spain

C. Albajar, J.F. de Trocóniz

Universidad de Oviedo, Instituto Universitario de Ciencias y Tecnologías Espaciales de Asturias (ICTEA), Oviedo, Spain

B. Alvarez Gonzalez, J. Cuevas, C. Erice, J. Fernandez Menendez, S. Folgueras, I. Gonzalez Caballero, J.R. González Fernández, E. Palencia Cortezon, V. Rodríguez Bouza, S. Sanchez Cruz

Instituto de Física de Cantabria (IFCA), CSIC-Universidad de Cantabria, Santander, Spain

I.J. Cabrillo, A. Calderon, B. Chazin Quero, J. Duarte Campderros, M. Fernandez, P.J. Fernández Manteca, A. García Alonso, G. Gomez, C. Martinez Rivero, P. Martinez Ruiz del Arbol, F. Matorras, J. Piedra Gomez, C. Prieels, T. Rodrigo, A. Ruiz-Jimeno, L. Russo $^{45}$, L. Scodellaro, N. Trevisani, I. Vila, J.M. Vizan Garcia

University of Colombo, Colombo, Sri Lanka

K. Malagalage

University of Ruhuna, Department of Physics, Matara, Sri Lanka

W.G.D. Dharmaratna, N. Wickramage 


\section{CERN, European Organization for Nuclear Research, Geneva, Switzerland}

D. Abbaneo, B. Akgun, E. Auffray, G. Auzinger, J. Baechler, P. Baillon, A.H. Ball, D. Barney, J. Bendavid, M. Bianco, A. Bocci, E. Bossini, C. Botta, E. Brondolin, T. Camporesi, A. Caratelli, G. Cerminara, E. Chapon, G. Cucciati, D. d'Enterria, A. Dabrowski, N. Daci, V. Daponte, A. David, O. Davignon, A. De Roeck, N. Deelen, M. Deile, M. Dobson, M. Dünser, N. Dupont, A. Elliott-Peisert, F. Fallavollita ${ }^{46}$, D. Fasanella, G. Franzoni, J. Fulcher, W. Funk, S. Giani, D. Gigi, A. Gilbert, K. Gill, F. Glege, M. Gruchala, M. Guilbaud, D. Gulhan, J. Hegeman, C. Heidegger, Y. Iiyama, V. Innocente, P. Janot, O. Karacheban ${ }^{20}$, J. Kaspar, J. Kieseler, M. Krammer ${ }^{1}$, C. Lange, P. Lecoq, C. Lourenço, L. Malgeri, M. Mannelli, A. Massironi, F. Meijers, J.A. Merlin, S. Mersi, E. Meschi, F. Moortgat, M. Mulders, J. Ngadiuba, S. Nourbakhsh, S. Orfanelli, L. Orsini, F. Pantaleo ${ }^{17}$, L. Pape, E. Perez, M. Peruzzi, A. Petrilli, G. Petrucciani, A. Pfeiffer, M. Pierini, F.M. Pitters, D. Rabady, A. Racz, M. Rovere, H. Sakulin, C. Schäfer, C. Schwick, M. Selvaggi, A. Sharma, P. Silva, W. Snoeys, P. Sphicas ${ }^{47}$, J. Steggemann, V.R. Tavolaro, D. Treille, A. Tsirou, A. Vartak, M. Verzetti, W.D. Zeuner

\section{Paul Scherrer Institut, Villigen, Switzerland}

L. Caminada ${ }^{48}$, K. Deiters, W. Erdmann, R. Horisberger, Q. Ingram, H.C. Kaestli, D. Kotlinski, U. Langenegger, T. Rohe, S.A. Wiederkehr

ETH Zurich - Institute for Particle Physics and Astrophysics (IPA), Zurich, Switzerland

M. Backhaus, P. Berger, N. Chernyavskaya, G. Dissertori, M. Dittmar, M. Donegà, C. Dorfer, T.A. Gómez Espinosa, C. Grab, D. Hits, T. Klijnsma, W. Lustermann, R.A. Manzoni, M. Marionneau, M.T. Meinhard, F. Micheli, P. Musella, F. Nessi-Tedaldi, F. Pauss, G. Perrin, L. Perrozzi, S. Pigazzini, M. Reichmann, C. Reissel, T. Reitenspiess, D. Ruini, D.A. Sanz Becerra, M. Schönenberger, L. Shchutska, M.L. Vesterbacka Olsson, R. Wallny, D.H. Zhu

\section{Universität Zürich, Zurich, Switzerland}

T.K. Aarrestad, C. Amsler ${ }^{49}$, D. Brzhechko, M.F. Canelli, A. De Cosa, R. Del Burgo, S. Donato, B. Kilminster, S. Leontsinis, V.M. Mikuni, I. Neutelings, G. Rauco, P. Robmann, D. Salerno, K. Schweiger, C. Seitz, Y. Takahashi, S. Wertz, A. Zucchetta

\section{National Central University, Chung-Li, Taiwan}

T.H. Doan, C.M. Kuo, W. Lin, A. Roy, S.S. Yu

\section{National Taiwan University (NTU), Taipei, Taiwan}

P. Chang, Y. Chao, K.F. Chen, P.H. Chen, W.-S. Hou, Y.y. Li, R.-S. Lu, E. Paganis, A. Psallidas, A. Steen

Chulalongkorn University, Faculty of Science, Department of Physics, Bangkok, Thailand

B. Asavapibhop, C. Asawatangtrakuldee, N. Srimanobhas, N. Suwonjandee 
Çukurova University, Physics Department, Science and Art Faculty, Adana, Turkey

A. Bat, F. Boran, S. Cerci $^{50}$, S. Damarseckin ${ }^{51}$, Z.S. Demiroglu, F. Dolek, C. Dozen, I. Dumanoglu, E. Eskut, G. Gokbulut, EmineGurpinar Guler ${ }^{52}$, Y. Guler, I. Hos ${ }^{53}$, C. Isik, E.E. Kangal ${ }^{54}$, O. Kara, A. Kayis Topaksu, U. Kiminsu, M. Oglakci, G. Onengut, K. Ozdemir ${ }^{55}$, S. Ozturk ${ }^{56}$, A.E. Simsek, U.G. Tok, S. Turkcapar, I.S. Zorbakir, C. Zorbilmez

Middle East Technical University, Physics Department, Ankara, Turkey

B. Isildak ${ }^{57}$, G. Karapinar ${ }^{58}$, M. Yalvac

Bogazici University, Istanbul, Turkey

I.O. Atakisi, E. Gülmez, M. Kaya ${ }^{59}$, O. Kaya ${ }^{60}$, B. Kaynak, Ö. Özçelik, S. Tekten, E.A. Yetkin ${ }^{61}$

Istanbul Technical University, Istanbul, Turkey

A. Cakir, K. Cankocak, Y. Komurcu, S. Sen ${ }^{62}$

Istanbul University, Istanbul, Turkey

S. Ozkorucuklu

Institute for Scintillation Materials of National Academy of Science of Ukraine, Kharkov, Ukraine

B. Grynyov

National Scientific Center, Kharkov Institute of Physics and Technology, Kharkov, Ukraine

L. Levchuk

University of Bristol, Bristol, United Kingdom

F. Ball, E. Bhal, S. Bologna, J.J. Brooke, D. Burns, E. Clement, D. Cussans, H. Flacher,

J. Goldstein, G.P. Heath, H.F. Heath, L. Kreczko, S. Paramesvaran, B. Penning,

T. Sakuma, S. Seif El Nasr-Storey, D. Smith, V.J. Smith, J. Taylor, A. Titterton

Rutherford Appleton Laboratory, Didcot, United Kingdom

K.W. Bell, A. Belyaev ${ }^{63}$, C. Brew, R.M. Brown, D. Cieri, D.J.A. Cockerill, J.A. Coughlan,

K. Harder, S. Harper, J. Linacre, K. Manolopoulos, D.M. Newbold, E. Olaiya, D. Petyt,

T. Reis, T. Schuh, C.H. Shepherd-Themistocleous, A. Thea, I.R. Tomalin, T. Williams, W.J. Womersley

\section{Imperial College, London, United Kingdom}

R. Bainbridge, P. Bloch, J. Borg, S. Breeze, O. Buchmuller, A. Bundock, GurpreetSingh CHAHAL ${ }^{64}$, D. Colling, P. Dauncey, G. Davies, M. Della Negra, R. Di Maria, P. Everaerts, G. Hall, G. Iles, T. James, M. Komm, C. Laner, L. Lyons, A.-M. Magnan, S. Malik, A. Martelli, V. Milosevic, J. $\mathrm{Nash}^{65}$, V. Palladino, M. Pesaresi, D.M. Raymond, A. Richards, A. Rose, E. Scott, C. Seez, A. Shtipliyski, M. Stoye, T. Strebler, S. Summers, A. Tapper, K. Uchida, T. Virdee ${ }^{17}$, N. Wardle, D. Winterbottom, J. Wright, A.G. Zecchinelli, S.C. Zenz 
Brunel University, Uxbridge, United Kingdom

J.E. Cole, P.R. Hobson, A. Khan, P. Kyberd, C.K. Mackay, A. Morton, I.D. Reid, L. Teodorescu, S. Zahid

Baylor University, Waco, U.S.A.

K. Call, J. Dittmann, K. Hatakeyama, C. Madrid, B. McMaster, N. Pastika, C. Smith

Catholic University of America, Washington, DC, U.S.A.

R. Bartek, A. Dominguez, R. Uniyal

The University of Alabama, Tuscaloosa, U.S.A.

A. Buccilli, S.I. Cooper, C. Henderson, P. Rumerio, C. West

Boston University, Boston, U.S.A.

D. Arcaro, T. Bose, Z. Demiragli, D. Gastler, S. Girgis, D. Pinna, C. Richardson, J. Rohlf,

D. Sperka, I. Suarez, L. Sulak, D. Zou

Brown University, Providence, U.S.A.

G. Benelli, B. Burkle, X. Coubez, D. Cutts, Y.t. Duh, M. Hadley, J. Hakala, U. Heintz, J.M. $\operatorname{Hogan}^{66}$, K.H.M. Kwok, E. Laird, G. Landsberg, J. Lee, Z. Mao, M. Narain, S. Sagir ${ }^{67}$, R. Syarif, E. Usai, D. Yu

University of California, Davis, Davis, U.S.A.

R. Band, C. Brainerd, R. Breedon, M. Calderon De La Barca Sanchez, M. Chertok, J. Conway, R. Conway, P.T. Cox, R. Erbacher, C. Flores, G. Funk, F. Jensen, W. Ko, O. Kukral, R. Lander, M. Mulhearn, D. Pellett, J. Pilot, M. Shi, D. Stolp, D. Taylor,

K. Tos, M. Tripathi, Z. Wang, F. Zhang

University of California, Los Angeles, U.S.A.

M. Bachtis, C. Bravo, R. Cousins, A. Dasgupta, A. Florent, J. Hauser, M. Ignatenko, N. Mccoll, W.A. Nash, S. Regnard, D. Saltzberg, C. Schnaible, B. Stone, V. Valuev

University of California, Riverside, Riverside, U.S.A.

K. Burt, R. Clare, J.W. Gary, S.M.A. Ghiasi Shirazi, G. Hanson, G. Karapostoli, E. Kennedy, O.R. Long, M. Olmedo Negrete, M.I. Paneva, W. Si, L. Wang, H. Wei, S. Wimpenny, B.R. Yates, Y. Zhang

University of California, San Diego, La Jolla, U.S.A.

J.G. Branson, P. Chang, S. Cittolin, M. Derdzinski, R. Gerosa, D. Gilbert, B. Hashemi, D. Klein, V. Krutelyov, J. Letts, M. Masciovecchio, S. May, S. Padhi, M. Pieri, V. Sharma, M. Tadel, F. Würthwein, A. Yagil, G. Zevi Della Porta

University of California, Santa Barbara - Department of Physics, Santa Barbara, U.S.A.

N. Amin, R. Bhandari, C. Campagnari, M. Citron, V. Dutta, M. Franco Sevilla, L. Gouskos, J. Incandela, B. Marsh, H. Mei, A. Ovcharova, H. Qu, J. Richman, U. Sarica, D. Stuart, S. Wang, J. Yoo 
California Institute of Technology, Pasadena, U.S.A.

D. Anderson, A. Bornheim, O. Cerri, I. Dutta, J.M. Lawhorn, N. Lu, J. Mao, H.B. Newman, T.Q. Nguyen, J. Pata, M. Spiropulu, J.R. Vlimant, S. Xie, Z. Zhang, R.Y. Zhu

Carnegie Mellon University, Pittsburgh, U.S.A.

M.B. Andrews, T. Ferguson, T. Mudholkar, M. Paulini, M. Sun, I. Vorobiev, M. Weinberg

University of Colorado Boulder, Boulder, U.S.A.

J.P. Cumalat, W.T. Ford, A. Johnson, E. MacDonald, T. Mulholland, R. Patel, A. Perloff, K. Stenson, K.A. Ulmer, S.R. Wagner

Cornell University, Ithaca, U.S.A.

J. Alexander, J. Chaves, Y. Cheng, J. Chu, A. Datta, A. Frankenthal, K. Mcdermott, N. Mirman, J.R. Patterson, D. Quach, A. Rinkevicius ${ }^{68}$, A. Ryd, S.M. Tan, Z. Tao, J. Thom, P. Wittich, M. Zientek

Fermi National Accelerator Laboratory, Batavia, U.S.A.

S. Abdullin, M. Albrow, M. Alyari, G. Apollinari, A. Apresyan, A. Apyan, S. Banerjee, L.A.T. Bauerdick, A. Beretvas, J. Berryhill, P.C. Bhat, K. Burkett, J.N. Butler, A. Canepa, G.B. Cerati, H.W.K. Cheung, F. Chlebana, M. Cremonesi, J. Duarte, V.D. Elvira, J. Freeman, Z. Gecse, E. Gottschalk, L. Gray, D. Green, S. Grünendahl, O. Gutsche, AllisonReinsvold Hall, J. Hanlon, R.M. Harris, S. Hasegawa, R. Heller, J. Hirschauer, B. Jayatilaka, S. Jindariani, M. Johnson, U. Joshi, B. Klima, M.J. Kortelainen, B. Kreis, S. Lammel, J. Lewis, D. Lincoln, R. Lipton, M. Liu, T. Liu, J. Lykken, K. Maeshima, J.M. Marraffino, D. Mason, P. McBride, P. Merkel, S. Mrenna, S. Nahn, V. O’Dell, V. Papadimitriou, K. Pedro, C. Pena, G. Rakness, F. Ravera, L. Ristori, B. Schneider, E. Sexton-Kennedy, N. Smith, A. Soha, W.J. Spalding, L. Spiegel, S. Stoynev, J. Strait, N. Strobbe, L. Taylor, S. Tkaczyk, N.V. Tran, L. Uplegger, E.W. Vaandering, C. Vernieri, M. Verzocchi, R. Vidal, M. Wang, H.A. Weber

University of Florida, Gainesville, U.S.A.

D. Acosta, P. Avery, P. Bortignon, D. Bourilkov, A. Brinkerhoff, L. Cadamuro, A. Carnes, V. Cherepanov, D. Curry, F. Errico, R.D. Field, S.V. Gleyzer, B.M. Joshi, M. Kim, J. Konigsberg, A. Korytov, K.H. Lo, P. Ma, K. Matchev, N. Menendez, G. Mitselmakher, D. Rosenzweig, K. Shi, J. Wang, S. Wang, X. Zuo

Florida International University, Miami, U.S.A.

Y.R. Joshi

Florida State University, Tallahassee, U.S.A.

T. Adams, A. Askew, S. Hagopian, V. Hagopian, K.F. Johnson, R. Khurana, T. Kolberg, G. Martinez, T. Perry, H. Prosper, C. Schiber, R. Yohay, J. Zhang

Florida Institute of Technology, Melbourne, U.S.A.

M.M. Baarmand, V. Bhopatkar, M. Hohlmann, D. Noonan, M. Rahmani, M. Saunders, F. Yumiceva 
University of Illinois at Chicago (UIC), Chicago, U.S.A.

M.R. Adams, L. Apanasevich, D. Berry, R.R. Betts, R. Cavanaugh, X. Chen, S. Dittmer, O. Evdokimov, C.E. Gerber, D.A. Hangal, D.J. Hofman, K. Jung, C. Mills, T. Roy, M.B. Tonjes, N. Varelas, H. Wang, X. Wang, Z. Wu

The University of Iowa, Iowa City, U.S.A.

M. Alhusseini, B. Bilki ${ }^{52}$, W. Clarida, K. Dilsiz ${ }^{69}$, S. Durgut, R.P. Gandrajula, M. Haytmyradov, V. Khristenko, O.K. Köseyan, J.-P. Merlo, A. Mestvirishvili ${ }^{70}$, A. Moeller, J. Nachtman, H. Ogul ${ }^{71}$, Y. Onel, F. Ozok ${ }^{72}$, A. Penzo, C. Snyder, E. Tiras, J. Wetzel

Johns Hopkins University, Baltimore, U.S.A.

B. Blumenfeld, A. Cocoros, N. Eminizer, D. Fehling, L. Feng, A.V. Gritsan, W.T. Hung, P. Maksimovic, J. Roskes, M. Swartz, M. Xiao

The University of Kansas, Lawrence, U.S.A.

C. Baldenegro Barrera, P. Baringer, A. Bean, S. Boren, J. Bowen, A. Bylinkin, T. Isidori, S. Khalil, J. King, G. Krintiras, A. Kropivnitskaya, C. Lindsey, D. Majumder, W. Mcbrayer, N. Minafra, M. Murray, C. Rogan, C. Royon, S. Sanders, E. Schmitz, J.D. Tapia Takaki, Q. Wang, J. Williams, G. Wilson

Kansas State University, Manhattan, U.S.A.

S. Duric, A. Ivanov, K. Kaadze, D. Kim, Y. Maravin, D.R. Mendis, T. Mitchell, A. Modak, A. Mohammadi

Lawrence Livermore National Laboratory, Livermore, U.S.A.

F. Rebassoo, D. Wright

University of Maryland, College Park, U.S.A.

A. Baden, O. Baron, A. Belloni, S.C. Eno, Y. Feng, N.J. Hadley, S. Jabeen, G.Y. Jeng, R.G. Kellogg, J. Kunkle, A.C. Mignerey, S. Nabili, F. Ricci-Tam, M. Seidel, Y.H. Shin, A. Skuja, S.C. Tonwar, K. Wong

Massachusetts Institute of Technology, Cambridge, U.S.A.

D. Abercrombie, B. Allen, A. Baty, R. Bi, S. Brandt, W. Busza, I.A. Cali, M. D'Alfonso,

G. Gomez Ceballos, M. Goncharov, P. Harris, D. Hsu, M. Hu, M. Klute, D. Kovalskyi, Y.-J. Lee, P.D. Luckey, B. Maier, A.C. Marini, C. Mcginn, C. Mironov, S. Narayanan, X. Niu, C. Paus, D. Rankin, C. Roland, G. Roland, Z. Shi, G.S.F. Stephans, K. Sumorok, K. Tatar, D. Velicanu, J. Wang, T.W. Wang, B. Wyslouch

University of Minnesota, Minneapolis, U.S.A.

A.C. Benvenuti ${ }^{\dagger}$, R.M. Chatterjee, A. Evans, S. Guts, P. Hansen, J. Hiltbrand, Sh. Jain, S. Kalafut, Y. Kubota, Z. Lesko, J. Mans, R. Rusack, M.A. Wadud

University of Mississippi, Oxford, U.S.A.

J.G. Acosta, S. Oliveros

University of Nebraska-Lincoln, Lincoln, U.S.A.

K. Bloom, D.R. Claes, C. Fangmeier, L. Finco, F. Golf, R. Gonzalez Suarez, R. Kamalieddin, I. Kravchenko, J.E. Siado, G.R. Snow, B. Stieger 
State University of New York at Buffalo, Buffalo, U.S.A.

G. Agarwal, C. Harrington, I. Iashvili, A. Kharchilava, C. Mclean, D. Nguyen, A. Parker, J. Pekkanen, S. Rappoccio, B. Roozbahani

Northeastern University, Boston, U.S.A.

G. Alverson, E. Barberis, C. Freer, Y. Haddad, A. Hortiangtham, G. Madigan, D.M. Morse,

T. Orimoto, L. Skinnari, A. Tishelman-Charny, T. Wamorkar, B. Wang, A. Wisecarver,

D. Wood

Northwestern University, Evanston, U.S.A.

S. Bhattacharya, J. Bueghly, T. Gunter, K.A. Hahn, N. Odell, M.H. Schmitt, K. Sung, M. Trovato, M. Velasco

University of Notre Dame, Notre Dame, U.S.A.

R. Bucci, N. Dev, R. Goldouzian, M. Hildreth, K. Hurtado Anampa, C. Jessop, D.J. Karmgard, K. Lannon, W. Li, N. Loukas, N. Marinelli, I. Mcalister, F. Meng, C. Mueller, Y. Musienko ${ }^{36}$, M. Planer, R. Ruchti, P. Siddireddy, G. Smith, S. Taroni, M. Wayne, A. Wightman, M. Wolf, A. Woodard

The Ohio State University, Columbus, U.S.A.

J. Alimena, B. Bylsma, L.S. Durkin, S. Flowers, B. Francis, C. Hill, W. Ji, A. Lefeld, T.Y. Ling, B.L. Winer

Princeton University, Princeton, U.S.A.

S. Cooperstein, G. Dezoort, P. Elmer, J. Hardenbrook, N. Haubrich, S. Higginbotham, A. Kalogeropoulos, S. Kwan, D. Lange, M.T. Lucchini, J. Luo, D. Marlow, K. Mei, I. Ojalvo, J. Olsen, C. Palmer, P. Piroué, J. Salfeld-Nebgen, D. Stickland, C. Tully, Z. Wang

University of Puerto Rico, Mayaguez, U.S.A.

S. Malik, S. Norberg

Purdue University, West Lafayette, U.S.A.

A. Barker, V.E. Barnes, S. Das, L. Gutay, M. Jones, A.W. Jung, A. Khatiwada, B. Mahakud, D.H. Miller, G. Negro, N. Neumeister, C.C. Peng, S. Piperov, H. Qiu, J.F. Schulte, J. Sun, F. Wang, R. Xiao, W. Xie

Purdue University Northwest, Hammond, U.S.A.

T. Cheng, J. Dolen, N. Parashar

Rice University, Houston, U.S.A.

K.M. Ecklund, S. Freed, F.J.M. Geurts, M. Kilpatrick, Arun Kumar, W. Li, B.P. Padley, R. Redjimi, J. Roberts, J. Rorie, W. Shi, A.G. Stahl Leiton, Z. Tu, A. Zhang

University of Rochester, Rochester, U.S.A.

A. Bodek, P. de Barbaro, R. Demina, J.L. Dulemba, C. Fallon, T. Ferbel, M. Galanti, A. Garcia-Bellido, J. Han, O. Hindrichs, A. Khukhunaishvili, E. Ranken, P. Tan, R. Taus 
Rutgers, The State University of New Jersey, Piscataway, U.S.A.

B. Chiarito, J.P. Chou, A. Gandrakota, Y. Gershtein, E. Halkiadakis, A. Hart, M. Heindl, E. Hughes, S. Kaplan, S. Kyriacou, I. Laflotte, A. Lath, R. Montalvo, K. Nash, M. Osherson, H. Saka, S. Salur, S. Schnetzer, D. Sheffield, S. Somalwar, R. Stone, S. Thomas, P. Thomassen

\section{University of Tennessee, Knoxville, U.S.A.}

H. Acharya, A.G. Delannoy, J. Heideman, G. Riley, S. Spanier

Texas A\&M University, College Station, U.S.A.

O. Bouhali ${ }^{73}$, A. Celik, M. Dalchenko, M. De Mattia, A. Delgado, S. Dildick, R. Eusebi, J. Gilmore, T. Huang, T. Kamon ${ }^{74}$, S. Luo, D. Marley, R. Mueller, D. Overton, L. Perniè, D. Rathjens, A. Safonov

\section{Texas Tech University, Lubbock, U.S.A.}

N. Akchurin, J. Damgov, F. De Guio, S. Kunori, K. Lamichhane, S.W. Lee, T. Mengke, S. Muthumuni, T. Peltola, S. Undleeb, I. Volobouev, Z. Wang, A. Whitbeck

\section{Vanderbilt University, Nashville, U.S.A.}

S. Greene, A. Gurrola, R. Janjam, W. Johns, C. Maguire, A. Melo, H. Ni, K. Padeken, F. Romeo, P. Sheldon, S. Tuo, J. Velkovska, M. Verweij

\section{University of Virginia, Charlottesville, U.S.A.}

M.W. Arenton, P. Barria, B. Cox, G. Cummings, R. Hirosky, M. Joyce, A. Ledovskoy, C. Neu, B. Tannenwald, Y. Wang, E. Wolfe, F. Xia

\section{Wayne State University, Detroit, U.S.A.}

R. Harr, P.E. Karchin, N. Poudyal, J. Sturdy, P. Thapa, S. Zaleski

\section{University of Wisconsin - Madison, Madison, WI, U.S.A.}

J. Buchanan, C. Caillol, D. Carlsmith, S. Dasu, I. De Bruyn, L. Dodd, F. Fiori, C. Galloni, B. Gomber ${ }^{75}$, M. Herndon, A. Hervé, U. Hussain, P. Klabbers, A. Lanaro, A. Loeliger, K. Long, R. Loveless, J. Madhusudanan Sreekala, T. Ruggles, A. Savin, V. Sharma, W.H. Smith, D. Teague, S. Trembath-reichert, N. Woods

\section{$\dagger$ : Deceased}

1: Also at Vienna University of Technology, Vienna, Austria

2: Also at IRFU, CEA, Université Paris-Saclay, Gif-sur-Yvette, France

3: Also at Universidade Estadual de Campinas, Campinas, Brazil

4: Also at Federal University of Rio Grande do Sul, Porto Alegre, Brazil

5: Also at UFMS, Nova Andradina, Brazil

6: Also at Universidade Federal de Pelotas, Pelotas, Brazil

7: Also at Université Libre de Bruxelles, Bruxelles, Belgium

8: Also at University of Chinese Academy of Sciences, Beijing, China

9: Also at Institute for Theoretical and Experimental Physics named by A.I. Alikhanov of NRC 'Kurchatov Institute', Moscow, Russia

10: Also at Joint Institute for Nuclear Research, Dubna, Russia

11: Also at Suez University, Suez, Egypt

12: Now at British University in Egypt, Cairo, Egypt 
13: Also at Purdue University, West Lafayette, U.S.A.

14: Also at Université de Haute Alsace, Mulhouse, France

15: Also at Tbilisi State University, Tbilisi, Georgia

16: Also at Erzincan Binali Yildirim University, Erzincan, Turkey

17: Also at CERN, European Organization for Nuclear Research, Geneva, Switzerland

18: Also at RWTH Aachen University, III. Physikalisches Institut A, Aachen, Germany

19: Also at University of Hamburg, Hamburg, Germany

20: Also at Brandenburg University of Technology, Cottbus, Germany

21: Also at Institute of Physics, University of Debrecen, Debrecen, Hungary, Debrecen, Hungary

22: Also at Institute of Nuclear Research ATOMKI, Debrecen, Hungary

23: Also at MTA-ELTE Lendület CMS Particle and Nuclear Physics Group, Eötvös Loránd University, Budapest, Hungary, Budapest, Hungary

24: Also at IIT Bhubaneswar, Bhubaneswar, India, Bhubaneswar, India

25: Also at Institute of Physics, Bhubaneswar, India

26: Also at Shoolini University, Solan, India

27: Also at University of Visva-Bharati, Santiniketan, India

28: Also at Isfahan University of Technology, Isfahan, Iran

29: Also at Italian National Agency for New Technologies, Energy and Sustainable Economic Development, Bologna, Italy

30: Also at Centro Siciliano di Fisica Nucleare e di Struttura Della Materia, Catania, Italy

31: Also at Scuola Normale e Sezione dell'INFN, Pisa, Italy

32: Also at Riga Technical University, Riga, Latvia, Riga, Latvia

33: Also at Malaysian Nuclear Agency, MOSTI, Kajang, Malaysia

34: Also at Consejo Nacional de Ciencia y Tecnología, Mexico City, Mexico

35: Also at Warsaw University of Technology, Institute of Electronic Systems, Warsaw, Poland

36: Also at Institute for Nuclear Research, Moscow, Russia

37: Now at National Research Nuclear University 'Moscow Engineering Physics Institute' (MEPhI), Moscow, Russia

38: Also at St. Petersburg State Polytechnical University, St. Petersburg, Russia

39: Also at University of Florida, Gainesville, U.S.A.

40: Also at Imperial College, London, United Kingdom

41: Also at P.N. Lebedev Physical Institute, Moscow, Russia

42: Also at California Institute of Technology, Pasadena, U.S.A.

43: Also at Budker Institute of Nuclear Physics, Novosibirsk, Russia

44: Also at Faculty of Physics, University of Belgrade, Belgrade, Serbia

45: Also at Università degli Studi di Siena, Siena, Italy

46: Also at INFN Sezione di Pavia ${ }^{a}$, Università di Pavia ${ }^{b}$, Pavia, Italy, Pavia, Italy

47: Also at National and Kapodistrian University of Athens, Athens, Greece

48: Also at Universität Zürich, Zurich, Switzerland

49: Also at Stefan Meyer Institute for Subatomic Physics, Vienna, Austria, Vienna, Austria

50: Also at Adiyaman University, Adiyaman, Turkey

51: Also at Şrnak University, Sirnak, Turkey

52: Also at Beykent University, Istanbul, Turkey, Istanbul, Turkey

53: Also at Istanbul Aydin University, Application and Research Center for Advanced Studies (App. \& Res. Cent. for Advanced Studies), Istanbul, Turkey

54: Also at Mersin University, Mersin, Turkey

55: Also at Piri Reis University, Istanbul, Turkey

56: Also at Gaziosmanpasa University, Tokat, Turkey 
57: Also at Ozyegin University, Istanbul, Turkey

58: Also at Izmir Institute of Technology, Izmir, Turkey

59: Also at Marmara University, Istanbul, Turkey

60: Also at Kafkas University, Kars, Turkey

61: Also at Istanbul Bilgi University, Istanbul, Turkey

62: Also at Hacettepe University, Ankara, Turkey

63: Also at School of Physics and Astronomy, University of Southampton, Southampton, United Kingdom

64: Also at IPPP Durham University, Durham, United Kingdom

65: Also at Monash University, Faculty of Science, Clayton, Australia

66: Also at Bethel University, St. Paul, Minneapolis, U.S.A., St. Paul, U.S.A.

67: Also at Karamanoğlu Mehmetbey University, Karaman, Turkey

68: Also at Vilnius University, Vilnius, Lithuania

69: Also at Bingol University, Bingol, Turkey

70: Also at Georgian Technical University, Tbilisi, Georgia

71: Also at Sinop University, Sinop, Turkey

72: Also at Mimar Sinan University, Istanbul, Istanbul, Turkey

73: Also at Texas A\&M University at Qatar, Doha, Qatar

74: Also at Kyungpook National University, Daegu, Korea, Daegu, Korea

75: Also at University of Hyderabad, Hyderabad, India 